G. van der Geer and K. Ueno

Nagoya Math. J.

Vol. 88 (1982), 17-53

\title{
FAMILIES OF ABELIAN SURFACES WITH REAL MULTIPLICATION OVER HILBERT MODULAR SURFACES
}

\author{
G. VAN DER GEER AND K. UENO
}

\section{§0. Introduction}

Around the beginning of this century G. Humbert ([9]) made a detailed study of the properties of compact complex surfaces which can be parametrized by singular abelian functions. A surface parametrized by singular abelian functions is the image under a holomorphic map of a singular abelian surface (i.e. an abelian surface whose endomorphism ring is larger than the ring of rational integers). Humbert showed that the periods of a singular abelian surface satisfy a quadratic relation with integral coefficients and he constructed an invariant $D$ of such a relation with respect to the action of the integral symplectic group on the periods. A few years later Hecke ([6]) made the connection with real quadratic fields by showing that the quotient of $\mathscr{H} \times \mathscr{H}_{-}$(the product of the complex upper and lower half plane) by the action of the symmetric Hilbert modular group of the field $Q(\sqrt{D})$ is the moduli space of singular abelian surfaces whose periods satisfy a relation with invariant $D$. The Hilbert modular surfaces have attracted new attention since Hirzebruch succeeded in resolving their singularities ([7]). This has resulted in a detailed knowledge of the geometry of these surfaces.

On the other hand, Mumford c.s. developed a compactification theory based on toroidal embeddings and this theory is an effective tool for the study of the degeneration of abelian varieties. Namikawa applied it to the quotient of the Siegel upper half space by the integral symplectic group to obtain a new compactification of this quotient and constructed degenerating abelian varieties over the compactified quotient. These

Received August 9, 1979.

Both authors were partially supported by the S.F.B. 40 "Theoretische Mathematik" at Bonn. 
recent developments have motivated us to study complex analytic families of abelian surfaces over Hilbert modular surfaces.

The composition of this paper is as follows. After having explained the meaning of the Hilbert modular surfaces as moduli spaces of abelian surfaces in section 1 , we recall in section 2 some notions from the theory of torus embeddings and we describe Hirzebruch's resolution of the cusps of Hilbert modular surfaces in section 3 . Section 4 is devoted to the construction of two compactifications, $\mathscr{A}_{\frac{1}{2}}$ and $\tilde{\mathscr{A}}_{\frac{1}{2}}$, of a (universal) family of polarised abelian surfaces over a Hilbert modular surface. Here we assume that the reader has some acquaintance with the construction of degenerating abelian varieties by means of toroidal embeddings (cf. Mumford [11] or Namikawa [13]). The first compactification $\mathscr{A}_{\frac{1}{2}}$ (the half period compactification) is singular; it seems to be the right compactification for the study of the degeneration of singular Kummer surfaces. For the construction of a non-singular compactification $\tilde{\mathscr{A}}_{\frac{1}{2}}$ we are indebted to $\mathrm{Y}$. Namikawa, who suggested to us the division process as explained in 4.8. In section 5 we prove that $\mathscr{A}_{\frac{1}{2}}$ and $\tilde{\mathscr{A}}_{\frac{1}{2}}$ are simply connected. Holomorphic differential forms on a toroidal compactification are described in section 6. In section 7 we express the arithmetic genus of such a compactification in terms of the volume of the Hilbert modular surface. In the last section we give a proof for the fact that a certain family of abelian varieties over a (compact) curve has only finitely many holomorphic sections and we apply it to obtain the number of sections of $\mathscr{A}_{\frac{1}{2}}$ and $\tilde{\mathscr{A}}_{\frac{1}{2}}$.

The authors would like to express their gratitude to Y. Namikawa for communicating to them the idea on which the non-singular compactification is based. They also want to thank D. Zagier for some useful comments during the preparation of this paper.

\section{Notation}

$\mathcal{O}^{*}:$ dual order of $\mathcal{O}$ with respect to the Trace. $x \rightarrow x^{(i)}(i=1,2)$ denotes the two real embeddings of an element $x$ of a real quadratic field. If $V_{1} \oplus V_{2}$ is an eigen decomposition of a vector space $V$ such that $x \in \mathcal{O}$ acts on $V_{i}$ by multiplication with $x^{(i)}$, then $\operatorname{Tr} x v=x^{(1)} v_{1}+x^{(2)} v_{2}$ for $v=$ $\left(v_{1}, v_{2}\right)$.

\section{§1. Abelian surfaces with real multiplication}

1.1. Let $\mathcal{O}$ be a maximal order in a real quadratic field $K$ and let $\mathfrak{A}$ 
be an invertible $\mathcal{O}$-module of rank 1 contained in $\mathcal{O}^{*}$, the $Z$-dual of $\mathcal{O}$ with respect to the trace. We define a $Z$-valued form $E_{\mathcal{A}}$ on the projective $\mathcal{O}_{-}$ module $\mathscr{M}=\mathcal{O} \oplus \mathfrak{A}$ by

$$
E_{\mathcal{M}}\left(\left(\alpha_{1}, \beta_{1}\right),\left(\alpha_{2}, \beta_{2}\right)\right)=\operatorname{Tr}_{K \mid \boldsymbol{Q}}\left(\alpha_{1} \beta_{2}-\alpha_{2} \beta_{1}\right)
$$

for $\left(\alpha_{i}, \beta_{i}\right) \in \mathcal{O} \oplus \mathfrak{U}$.

Let $\mathscr{H}$ be the complex upper half plane. To a point $z=\left(z_{1}, z_{2}\right) \in \mathscr{H}^{2}$ $=\mathscr{H} \times \mathscr{H}$ we associate the embedding $\mathscr{L}_{z}: K \oplus K \rightarrow V=C^{2}$ given by

$$
(\alpha, \beta) \longrightarrow\left(\alpha^{(1)} z_{1}+\beta^{(1)}, \alpha^{(2)} z_{2}+\beta^{(2)}\right),
$$

where $x \rightarrow x^{(i)}(i=1,2)$ denote the two real embeddings of $K$. This embedding determines a lattice $\mathscr{L}_{z}(\mathscr{M})$ in $V$. Transferring $E_{\mathcal{L}}$ to $\mathscr{L}_{z}(\mathscr{M})$ by $\mathscr{L}_{z}$ and extending it $\boldsymbol{R}$-linearly we obtain an $\boldsymbol{R}$-valued $\boldsymbol{R}$-bilinear form $E_{\mathcal{H}, z}: V \times V \rightarrow \boldsymbol{R}$. We easily compute that

$$
E_{\varkappa, z}(\zeta, \eta)=\sum_{j=1}^{2}\left(\operatorname{Im} z_{j}\right)^{-1} \operatorname{Im}\left(\zeta_{j} \bar{\eta}_{j}\right)
$$

for $\zeta=\left(\zeta_{1}, \zeta_{2}\right), \eta=\left(\eta_{1}, \eta_{2}\right) \in V$. In particular

$$
E_{\mathscr{\mu}, z}(i \zeta, i \eta)=E_{\mathscr{\mu}, z}(\zeta, \eta) \quad \text { and } \quad E_{\mathscr{\mu}, z}(i \eta, \eta)=\sum\left(\operatorname{Im} z_{j}\right)^{-1}\left|\eta_{j}\right|^{2} .
$$

Since $E_{\mathscr{k}, z}$ is integral on $\mathscr{L}_{z}(\mathscr{M})$ it defines a Riemann form on $V$ with respect to $\mathscr{L}_{z}(\mathscr{M})$. In this way the complex torus $V / \mathscr{L}_{z}(\mathscr{M})$ becomes a polarized abelian surface $A(z, \mathscr{M})$. Moreover, there is an injective homomorphism $m: \mathcal{O} \rightarrow$ End $(A(z, \mathscr{M}))$ given by

$$
m(\alpha):\left(\zeta_{1}, \zeta_{2}\right) \longrightarrow\left(\alpha^{(1)} \zeta_{1}, \alpha^{(2)} \zeta_{2}\right), \quad \alpha \in \mathcal{O} .
$$

1.2. Suppose that $T$ is a complex torus of dimension 2 and let $m: \mathcal{O}$ $\rightarrow$ End $(T)$ be a homomorphism sending 1 to Id. Set $V=\operatorname{Lie}(T), \mathscr{L}=$ $H_{1}(V, Z)$. Then $T=V / \mathscr{L}$ and $m$ gives a complex linear action of $\mathcal{O}$ on $V$ under which $\mathscr{L}$ is stable. We have an eigen space decomposition of $V$ as $V^{(1)} \oplus V^{(2)}\left(\operatorname{dim} V^{(i)}=1\right)$ such that $\alpha \in \mathcal{O}$ acts on $V^{(i)}$ by multiplication with $\alpha^{(i)} \subset \boldsymbol{R}$. Since $\mathcal{O}$ is a Dedekind ring, we can find an isomorphism $\varphi$ of the projective $\mathcal{O}$-module $\mathscr{L}$ of rank 2 with $\mathcal{O} \oplus \mathfrak{A}$ for some invertible $\mathcal{O}$-module $\mathfrak{A}$ of rank 1 . Choosing $\varphi$ determines an isomorphism of $V=$ $V^{(1)} \oplus V^{(2)}$ with $\boldsymbol{C} \oplus \boldsymbol{C}$ such that $(0, \beta) \in \mathcal{O} \oplus \mathfrak{X} \cong \mathscr{\overline { \varphi }} \subset V^{(1)} \oplus V^{(2)}$ maps to $\left(\beta^{(1)}, \beta^{(2)}\right)$. Then $(\alpha, 0)$ maps to $\left(\alpha^{(1)} z_{1}, \alpha^{(2)} z_{2}\right) \in C \oplus C$ with well-defined elements $\boldsymbol{z}_{i} \in \boldsymbol{C} \backslash \boldsymbol{R}$.

Replacing $\mathfrak{A}$ by $\lambda \mathfrak{U}$ replaces $\left(z_{1}, z_{2}\right)$ by $\left(\lambda^{(1)} z_{1}, \lambda^{(2)} z_{2}\right)$, so we may assume $z_{1}, z_{2} \in \mathscr{H}$. 
Thus $\left(z_{1}, z_{2}\right) \in \mathscr{H}^{2}$ and we get coordinates on $V$ with $\mathscr{L}=\mathscr{L}_{z}(\mathcal{O} \oplus \mathfrak{U})$ as in 1.1. Hence the construction of 1.1 shows that $T$ carries the structure of a polarized abelian variety with real multiplication by $\mathcal{O}$; however, this structure is not unique, but depends on the isomorphism $\varphi$.

1.3. Conversely, we assert that any polarization on $T$ arises in the manner described above for some $\varphi$. The Riemann form $E: \mathscr{L} \times \mathscr{L} \rightarrow Z$ extends to an alternating form $E: K \times K \rightarrow \boldsymbol{Q}$ and satisfies $E\left(x l_{1}, l_{2}\right)=$ $E\left(l_{1}, x l_{2}\right)$ for $x \in K$. The set of $x \in K$ for which the form $\left(l_{1}, l_{2}\right) \rightarrow E\left(x l_{1}, l_{2}\right)$ is $Z$-valued on $\mathscr{L} \times \mathscr{L}$ equals $\mathfrak{U}^{*}$ for some ideal $\mathfrak{U} \subset \mathcal{O}^{*}$. Then $\mathscr{L}$ is isomorphic as an $\mathcal{O}$-module to $\mathscr{M}=\mathcal{O} \oplus \mathfrak{U}$ in such a way that $E$ corresponds to $E_{\boldsymbol{N}}$. The different $\varphi$ 's with this property differ by the action of the group

$$
S L_{2}(\mathcal{O}, \mathfrak{U})=\left\{\left(\begin{array}{ll}
\alpha & \beta \\
\gamma & \delta
\end{array}\right) \in S L_{2}(K) \mid \alpha, \delta \in \mathcal{O}, \beta \in \mathfrak{A}, \gamma \in \mathfrak{X}^{-1}\right\}
$$

and they lead to points $z \in \mathscr{H}^{2}$ differing by the action of $S L_{2}(\mathcal{O}, \mathfrak{U})$ on $\mathscr{H}^{2}$ given by

$$
\left(z_{1}, z_{2}\right) \longrightarrow\left(\frac{\alpha^{(1)} z_{1}+\beta^{(1)}}{\gamma^{(1)} z_{1}+\delta^{(1)}}, \frac{\alpha^{(2)} z_{2}+\beta^{(2)}}{\gamma^{(2)} z_{2}+\delta^{(2)}}\right)
$$

Thus an abelian variety $A$ with multiplication by $\mathcal{O}$ and a given polarization determines uniquely an invertible $\mathcal{O}$-module $\mathfrak{U} \subset \mathcal{O}^{*}$ of rank 1 and a point $z \in \mathscr{H}^{2} / S L_{2}(\mathcal{O}, \mathfrak{O})$. Hence the coarse moduli space for polarized abelian surfaces with multiplication by $\mathcal{O}$ is the disjoint union of infinitely many components $\mathscr{H}^{2} / S L_{2}(\mathcal{O}, \mathfrak{U}), \mathfrak{A} \subset \mathcal{O}^{*}$ an invertible $\mathcal{O}$-module of rank 1.

Observe that the surfaces $\mathscr{H}^{2} / S L_{2}(\mathcal{O}, \mathfrak{X})$ and $\mathscr{H}^{2} / S L_{2}(\mathcal{O}, \lambda \mathscr{O})(\lambda \in K, \lambda \gg 0$ (totally positive)) are isomorphic via $\left(z_{1}, z_{2}\right) \rightarrow\left(\lambda^{(1)} z_{1}, \lambda^{(2)} z_{2}\right)$ but this changes the polarization of the corresponding abelian varieties. The coarse moduli space of abelian varieties with multiplication by $\mathcal{O}$ (without specified polarization) has $h^{+}$components, where $h^{+}$is the order of the group of narrow ideal classes of $\mathcal{O}$.

1.4. Consider an abelian surface $A$ with period matrix $T^{\prime}=(T D)$, where $T$ is a complex symmetric $2 \times 2$ matrix whose imaginary part is positive definite and $D$ is a diagonal matrix $\operatorname{diag}\left(d_{1}, d_{2}\right)$ with $d_{i} \in N, d_{1} \mid d_{2}$.

Suppose that $A$ admits complex multiplication. This means that

$$
M T^{\prime}=T^{\prime} S
$$


for some $M \in G L(2, C), M \notin Z \cdot I_{2}$ and some $\left.S \in M_{4}(Z)\right)$. The existence of complex multiplication implies conditions on $T$, namely

$$
T S_{12} D^{-1} T+D S_{22} D^{-1} T-T S_{11}-D S_{21}=0
$$

where we have written $S=\left(S_{i j}\right)_{1 \leq i, j \leq 2}$ with $S_{i j} \in M_{2}(Z)$. If we write $T=$ $\left(\begin{array}{ll}\tau_{1} & \tau_{2} \\ \tau_{2} & \tau_{3}\end{array}\right)$, we get relations of the form:

$$
a \tau_{1}+b \tau_{2}+c \tau_{3}+d\left(\tau_{2}^{2}-\tau_{1} \tau_{3}\right)+e=0,
$$

where the coefficients $a, b, \cdots, e$ are integers. We call such a relation between the $\tau_{i}$ a singular relation.

Principally polarized abelian surfaces whose period matrix $T^{\prime}=\left(T, I_{2}\right)$ satisfies a singular relation, were studied by Humbert ([9]). He defined $\Delta=b^{2}-4 a c-4 d e$ as the invariant of a singular relation and proved that $\mathrm{Sp}_{4}(Z)$ acts transitively on the set of singular relations with invariant $\Delta$. The invariant $\Delta$ is a positive integer which is congruent to 0 or $1(\bmod 4)$.

1.5. Let $\Delta$ be the discriminant of a real quadratic field $K$ and $\mathcal{O}$ the ring of integers of $K$. In his thesis ([6]) Hecke showed that one may embed $\mathscr{H} \times \mathscr{H}_{-}\left(\mathscr{H}_{-}:\right.$lower half plane of $C$ ) into the Siegel half space $\widetilde{S}_{2}$ in such a way that every point $T$ in the image satisfies a singular relation with invariant $\Delta$ and that the stabilizer in $S p_{4}(Z)$ of the image of $\mathscr{H} \times \mathscr{H}_{-}$is the symmetric Hilbert modular group (the extension of $S L_{2}(\mathcal{O})$ by the involution $\left.\left(z_{1}, z_{2}\right) \rightarrow\left(-z_{2},-z_{1}\right)\right)$. The embedding is given by

$$
\left(z_{1}, z_{2}\right) \longrightarrow \frac{1}{\sqrt{\Delta}}\left(\begin{array}{ll}
\operatorname{Tr} \mu_{1}^{2} \sqrt{\Delta} z & \operatorname{Tr} \mu_{1} \mu_{2} \sqrt{\Delta} z \\
\operatorname{Tr} \mu_{1} \mu_{2} \sqrt{\Delta} z & \operatorname{Tr} \mu_{2}^{2} \sqrt{\Delta} z
\end{array}\right)
$$

where $\mu_{1}, \mu_{2}$ is an oriented basis of the inverse different $\mathcal{O}^{*}$ (i.e. $\mu_{1}^{(1)} \mu_{2}^{(2)}-$ $\left.\mu_{2}^{(1)} \mu_{1}^{(2)}=+\sqrt{\bar{\Delta}}\right)$ and $\operatorname{Tr} \gamma z=\gamma^{(1)} z_{1}+\gamma^{(2)} z_{2}$. The map

$$
\left(z_{1}, z_{2}\right) \longrightarrow\left(\sqrt{\Delta} z_{1},-\sqrt{\Delta} z_{2}\right)
$$

defines an equivalence between the action of $S L_{2}(\mathcal{O})$ on $\mathscr{H} \times \mathscr{H}$ - and that of $S L_{2}\left(\mathcal{O}, \mathcal{O}^{*}\right)$ on $\mathscr{H}^{2}$.

If $\mathcal{O}$ has a unit of negative norm $\varepsilon$, then

$$
\left(z_{1}, z_{2}\right) \longrightarrow\left(\varepsilon z_{1}, \varepsilon^{\prime} z_{2}\right)
$$

(assume $\varepsilon>0$ ) identifies the action of $S L_{2}(\mathcal{O})$ on $\mathscr{H} \times \mathscr{H}_{-}$with that on $\mathscr{H}^{2}$.

1.6. We consider a generalization of Hecke's idea. Let $D$ be an elementary divisor matrix, 


$$
D=\left(\begin{array}{cc}
d_{1} & 0 \\
0 & d_{2}
\end{array}\right), \quad d_{1} \mid d_{2}, \quad d_{i} \in N
$$

We denote by $S p_{4}(Z, D)$ the paramodular group

$$
\left\{\left(\begin{array}{ll}
a & b \\
c & d
\end{array}\right) \in S p_{4}(Q) \mid a, \cdots, d \in M_{2}(Q), a, b D, D^{-1} c, D^{-1} d D \in M_{2}(Z)\right\} .
$$

By a modular embedding of a Hilbert modular group $\Gamma$ into $S p_{4}(Z, D)$ we mean a homomorphism

$$
\varphi: \Gamma \longrightarrow S_{4}(Z, D)
$$

and a holomorphic embedding

$$
\rho: \mathscr{H}^{2} \longrightarrow \widetilde{\complement}^{2}
$$

such that

i) $\varphi(\Gamma)$ is contained in the stabilizer of $\rho\left(\mathscr{H}^{2}\right)$,

ii) Siegel modular forms yield Hilbert modular forms by restriction to $\rho\left(\mathscr{H}^{2}\right)$.

The concept of modular embedding was studied by Hammond, Freitag and Schneider ([4], [3]).

1.7. We consider a special kind of modular embedding. For $z=\left(z_{1}, z_{2}\right)$ $\in \mathscr{H}^{2}$ we set $z^{*}=\left(\begin{array}{cc}z_{1} & 0 \\ 0 & z_{2}\end{array}\right)$. Similarly, for $\alpha \in K$ we set $\alpha^{*}=\left(\begin{array}{ll}\alpha^{(1)} & 0 \\ 0 & \alpha^{(2)}\end{array}\right)$.

Let $B \in G L(2, R)$. We define

$$
\rho_{B}: \mathscr{H}^{2} \longrightarrow \mathbb{\varsigma}^{2}
$$

by

$$
\rho_{B}(z)=B z^{* t} B
$$

and

$$
\varphi_{B}: \Gamma \longrightarrow \operatorname{Sp}_{4}(Z, D)
$$

by

$$
\varphi_{B}\left(\begin{array}{ll}
\alpha & \beta \\
\gamma & \delta
\end{array}\right)=\left(\begin{array}{cc}
B & 0 \\
0 & { }^{t} B^{-1}
\end{array}\right)\left(\begin{array}{cc}
\alpha^{*} & \beta^{*} \\
\gamma^{*} & \delta^{*}
\end{array}\right)\left(\begin{array}{cc}
B^{-1} & 0 \\
0 & { }^{t} B
\end{array}\right) .
$$

If $\Gamma=S L_{2}(\mathcal{O})$ and $\mathcal{O}$ is the maximal order in $K$, then $\left(\rho_{B}, \varphi_{B}\right)$ defines a modular embedding if and only if $B^{t} B D$ is unimodular and $B \alpha^{*} B^{-1}$ is integral for all $\alpha \in \mathcal{O}$. 
1.8. Call two modular embeddings $\left(\rho_{B}, \varphi_{B}\right)$ and $\left(\rho_{B^{\prime}}, \varphi_{B^{\prime}}\right)$ as in (1.9) equivalent if $B^{\prime}=U B$ with $U \in S L_{2}(Z)$ and $D U D^{-1} \in G L(2, Z)$. Freitag and Schneider ([3]) have proved that the equivalence classes of such modular embeddings of $S L_{2}(\mathcal{O})$ are in 1-1 correspondence with the decompositions of the inverse different $\mathcal{O}^{*}$ as $\rho \cdot \mathfrak{U} \cdot \mathfrak{B}$, where $\rho \gg 0$, and $\mathfrak{X}, \mathfrak{B}$ are fractional ideals with $\mathfrak{B} \subset \mathfrak{U}$ such that $\mathfrak{X} / \mathfrak{B} \cong Z / d_{1} Z \times Z / d_{2} Z$ as an abelian group. Here the decompositions $\mathcal{O}^{*}=\rho \cdot \mathfrak{U} \cdot \mathfrak{B}$ and $\mathcal{O}^{*}=\rho \alpha^{-2} \cdot \alpha \mathfrak{U} \cdot \alpha \mathfrak{B}$ are regarded as equivalent. In particular, a modular embedding of this type of $S L_{2}(\mathcal{O})$ in $S p_{4}(Z)$ (i.e. with $d_{1}=d_{2}=1$ ) is possible only if $\mathcal{O}^{*}=\rho \cdot \mathfrak{X}^{2}, \rho \gg 0$, i.e. if $\mathcal{O}^{*}$ is a square in the narrow ideal class group. On the other hand, there is always a modular embedding of $S L_{2}\left(\mathcal{O}, \mathcal{O}^{*}\right)$ in $S p_{4}(Z)$, namely the one given by $B=\left(\theta_{i}^{(j)}\right)$, where $\theta_{1}, \theta_{2}$ is a basis of $\mathcal{O}^{*}$ (this in fact holds for any order $\mathcal{O}$, not just the maximal order).

1.9. Let $z \in \mathscr{H}^{2}$ and let $\mathscr{M}=\mathcal{O} \oplus \mathfrak{A}$ be a projective $\mathcal{O}$-module of rank 2. On the abelian surface $A(z, \mathscr{M})$ we have the Riemann form $E$ (see 1.1). Then the polarization is given by the elementary divisors of the abelian group $\mathcal{O}^{*} / \mathfrak{U}=Z / d_{1} Z \times Z / d_{2} Z$; in particular, $A(z, \mathscr{M})$ has principal polarization only for $\mathfrak{A}=\mathcal{O}^{*}$.

The action of $S L_{2}(\mathcal{O})$ on $\mathscr{H} \times \mathscr{H}_{-}$is equivalent with that of $S L_{2}\left(\mathcal{O}, \mathcal{O}^{*}\right)$ on $\mathscr{H}^{2}$. If it is equivalent to that of $S L_{2}(\mathcal{O})$ on $\mathscr{H}^{2}$, then $S L_{2}(\mathcal{O})$ admits a modular embedding in $S p_{4}(Z)$, hence $\mathcal{O}^{*}=\rho \mathfrak{Y}^{2}$. Conversely, if $\mathcal{O}^{*}=\rho \mathfrak{U}^{2}$, then there is an equivalence between the actions of $S L_{2}(\mathcal{O})$ on $\mathscr{H} \times \mathscr{H}$ and $\mathscr{H} \times \mathscr{H}$ - given by conjugating $S L_{2}(\mathcal{O})$ into $S L_{2}\left(\mathcal{O}, \mathcal{O}^{*}\right)$ by an element $\left(\begin{array}{ll}a & b \\ c & d\end{array}\right) \in G L_{2}(K)$ with $(c, d)=\mathfrak{A}$ (cf. Hammond [5]).

1.10. Let $\Gamma$ be a congruence subgroup of $S L_{2}(\mathcal{O}, \mathfrak{Y})$. We define an action of $\mathscr{M}=\mathcal{O} \oplus \mathfrak{A}$ on $\mathscr{H}^{2} \times C^{2}$ by $\left(z_{1}, z_{2} ; \zeta_{1}, \zeta_{2}\right) \longrightarrow\left(z_{1}, z_{2} ; \zeta_{1}+\alpha^{(1)} z_{1}+\beta^{(1)}, \zeta_{2}+\alpha^{(2)} z_{2}+\beta^{(2)}\right) \quad((\alpha, \beta) \in \mathcal{O} \oplus \mathfrak{U})$. The Hilbert modular group $S L_{2}(\mathcal{O}, \mathfrak{U})$ acts on $\mathscr{H}^{2} \times C^{2}$ by

$$
\begin{aligned}
& \left(z_{1}, z_{2} ; \zeta_{1}, \zeta_{2}\right) \\
& \quad \longrightarrow\left(\frac{\alpha^{(1)} z_{1}+\beta^{(1)}}{\gamma^{(1)} z_{1}+\delta^{(1)}}, \frac{\alpha^{(2)} z_{2}+\beta^{(2)}}{\gamma^{(2)} z_{2}+\delta^{(2)}} ;\left(\gamma^{(1)} z_{1}+\delta^{(1)}\right)^{-1} \zeta_{1},\left(\gamma^{(2)} z_{2}+\delta^{(2)}\right)^{-1} \zeta_{2}\right)
\end{aligned}
$$

for $\left(\begin{array}{ll}\alpha & \beta \\ \gamma & \delta\end{array}\right) \in S L_{2}(\mathcal{O}, \mathfrak{X})$.

These two actions define an action of the semi-direct product $\Gamma \ltimes \mathscr{M}$ 
on $\mathscr{H}^{2} \times C^{2}$. It is properly discontinuous. Moreover, it is free if the action of $\Gamma$ on $\mathscr{H}^{2}$ is free. Let us assume this.

By taking the quotient we obtain a complex analytic family of abelian surfaces with (real) multiplication by $\mathcal{O}$. We shall write

$$
\pi: \mathscr{A}^{0}(\Gamma, \mathscr{M}) \longrightarrow \mathscr{H}^{2} / \Gamma
$$

or simply

$$
\pi: \mathscr{A}^{0} \longrightarrow \mathscr{H}^{2} / \Gamma
$$

for this family.

1.11. Let $(\rho, \varphi)$ be a modular embedding of $\Gamma$ into $S p_{4}(Z, D)$. Assume that $\rho(\Gamma)$ is contained in the principal congruence group of level $N$ of $S p_{4}(Z, D), N \geq 3$. Then $(\rho, \varphi)$ defines a family of polarized abelian surfaces over $\mathscr{H}^{2} / \Gamma$. Indeed the translations

$$
\begin{aligned}
& \mathfrak{S}_{2} \times C^{2} \longrightarrow \quad \mathfrak{S}_{2} \times C^{2} \\
& (\tau, \zeta) \longmapsto(\tau, \zeta+m \tau+n)
\end{aligned}
$$

for $m, n \in Z^{2}$ and the action of the paramodular group

$$
(\tau, \zeta) \longmapsto\left((A \tau+B)(C \tau+D)^{-1}, \zeta(C \tau+D)^{-1}\right)
$$

for $\left(\begin{array}{ll}A & B \\ C & D\end{array}\right) \in S p_{4}(Z, D)$ define an action of $S p_{4}(Z, D) \times Z^{2}$ on $\widetilde{S}_{2} \times C^{2}$.

The quotient $\widetilde{S}_{2} \times C^{2} / \Gamma(N) \times Z^{2}(\Gamma(N)$ the principal congruence subgroup of $S p_{4}(Z, D)$ of level $\left.N \geq 3\right)$ is a family of polarized abelian surfaces over $\mathfrak{S}_{2} / \Gamma(N)$. Its pull-back by means of $\rho$ is a family over $\mathscr{H}^{2} / \Gamma$. It coincides with a family constructed as in 1.10 if the modular embedding is as in 1.9 .

\section{§2. Toroidal embeddings}

2.0. In this section we recall the notions from the theory of toroidal embeddings (over $C$ ) which we need in the sequel. We refer to [10] and [1] for the general theory.

2.1. Consider $\mathscr{T}=\operatorname{Spec}\left(C\left[T_{1}, T_{1}^{-1}, \cdots, T_{n}, T_{n}^{-1}\right]\right)=G_{m}^{n}(C)$, the $n$-dimensional complex algebraic torus. We write

$$
M=\operatorname{Hom}\left(\boldsymbol{G}_{m}^{n}, \boldsymbol{G}_{m}\right) \quad \text { for the algebraic character group }
$$


and $N=\operatorname{Hom}\left(\boldsymbol{G}_{m}, \boldsymbol{G}_{m}^{n}\right)$ for the group of algebraic 1-parameter subgroups of $\mathscr{T}$. To each $r \in M$ we can associate canonically a section $\mathfrak{X}^{r} \in \Gamma\left(T, \mathcal{O}_{\mathscr{\sigma}}\right)$. There exists a natural pairing

$$
\langle,\rangle: M \times N \longrightarrow Z
$$

such that $\mathfrak{X}^{r}(\lambda(t))=t^{\langle r, \lambda\rangle}$ for all $t \in \mathscr{T}$.

We may view $M$ and $N$ as dual free abelian groups of rank $n$.

2.2. We shall write $\mathscr{T}$ as a product of its maximal compact torus and $N \otimes \boldsymbol{R}$,

Identify $N$ canonically with the fundamental group $\pi_{1}(\mathscr{T})$ of $\mathscr{T}$. This gives a canonical isomorphism of $\mathscr{T}$ with $N \otimes C / N$. Then $N \otimes R / N$ lies in $\mathscr{T}$ as the maximal compact torus and we have an isomorphism

$$
N \otimes C / N \longrightarrow(N \otimes R) / N \times(N \otimes R) .
$$

We denote by "ord" the projection $\mathscr{T} \rightarrow N \otimes R$ defined by taking the imaginary part.

2.3. A convex rational polyhedral cone $\sigma$ in $M_{R}=M \otimes R$ (or in $N_{R}$ $=N \otimes R)$ is a set

$$
\sigma=\left\{x \in M_{R}\left(\operatorname{resp} . N_{R}\right) \mid l_{i}(x) \geq 0 i=1, \cdots, k\right\},
$$

where the $l_{i}$ denote linear forms defined over $\boldsymbol{Q}$.

A rational partial polyhedral decomposition of $N_{R}$ consists of a collection $\Sigma=\left\{\sigma_{\alpha}\right\}$ of convex rational polyhedral cones such that

i) no $\sigma_{\alpha}$ contains a linear subspace,

ii) for $\sigma_{\alpha} \in \Sigma$ all faces of $\sigma_{\alpha}$ belong to $\Sigma$,

iii) the intersection of two different $\sigma_{\alpha}$ 's is a face of both $\sigma_{\alpha}$ and $\sigma_{\beta}$.

We define the dual cone $\hat{\sigma}$ of a convex rational polyhedral cone $\sigma$ in $N_{R}$ as

$$
\hat{\sigma}=\left\{m \in M_{R} \mid\langle m, n\rangle \geq 0 \text { for all } n \in \sigma\right\} .
$$

Then $\hat{\sigma}$ is a convex rational polyhedral cone in $M_{R}$.

2.4. An (affine) torus embedding of $\mathscr{T}$ is an (affine) variety containing $\mathscr{T}$ as an open subset together with an action of $\mathscr{T}$ which extends the action of $\mathscr{T}$ on itself given by the translations. To a semi-group $S \subset M$ we associate the subspace $C[S]$ of $\Gamma\left(\mathscr{T}, \mathcal{O}_{\mathcal{F}}\right)$ generated by $\mathfrak{X}^{r}, r \in S$. If $S$ generates $M$ as a group then $C[S] \subset \Gamma\left(\mathscr{T}, \mathcal{O}_{\mathscr{F}}\right)$ induces an affine torus 
embedding $\mathscr{T} \rightarrow \operatorname{Spec}(C[S])$. This procedure yields all affine torus embeddings of $\mathscr{T}$. Let $\sigma$ be a convex rational polyhedral cone in $N_{R}$ which contains no linear subspace. Then $\sigma$ defines a normal affine torus embedding

$$
\mathscr{X}_{\sigma}=\operatorname{Spec}(C[\hat{\sigma} \cap M]) .
$$

If $\Sigma=\left\{\sigma_{\alpha}\right\}$ is a rational partial polyhedral decomposition of $N_{R}$, the torus embeddings $\mathscr{X}_{\sigma_{\alpha}}$ can be glued together to form a normal torus embedding $\mathscr{X}_{\Sigma}$, which is locally of finite type.

There exists a bijection between the set of $\mathscr{T}$-orbits of $X_{\Sigma}$ and the set of convex rational polyhedral cones. All elements $\nu \in N$ in the relative interior $\sigma^{0}$ of $\sigma$ determine the same limit $\nu(0)=\lim _{t \rightarrow 0} \nu(t)$. It is the unique closed orbit $O(\sigma)$ of $\mathscr{X}_{\sigma}$. We have

$$
\operatorname{dim} \sigma+\operatorname{dim} O(\sigma)=\operatorname{dim} T,
$$

$\sigma_{\alpha} \subset \sigma_{\beta}$ if and only if $O^{\sigma \beta} \subset \overline{\left(O^{\sigma \alpha}\right)}$

2.5. A convex rational polyhedral cone in $N \otimes \boldsymbol{R}$ is said to be regular if it is the cone over a simplex in $N \otimes \boldsymbol{R}$ whose vertices are part of a $Z$-basis of $N$. A rational partial polyhedral decomposition $\Sigma$ is said to be regular if all $\sigma_{\alpha} \in \Sigma$ are regular. If $\Sigma$ is regular, then $\mathscr{X}_{\Sigma}$ is nonsingular.

A rational partial polyhedral decomposition is said to be projective if there exists a convex real-valued function $f$ on the convex hull of $\bigcup_{\sigma \in \Sigma} \sigma \subset N_{R}$ such that

i) the restriction of $f$ to $\sigma$ is linear for every $\sigma \in \Sigma$,

ii) $f$ is integral on $\left(\bigcup_{\sigma \in \Sigma} \sigma\right) \cap N$,

iii) each cone $\sigma \in \Sigma$ can be defined by

$$
\sigma=\left\{\nu \in N_{R} \mid f(\nu)=\lambda_{i, \sigma}(\nu), i=1, \cdots, k_{\sigma}\right\}
$$

where the $\lambda_{i, \sigma}$ are linear forms on $N_{\boldsymbol{R}}$ defined over $\boldsymbol{Q}$.

If $\Sigma$ is projective, the $\Gamma\left(\mathscr{X}_{\sigma}, \mathcal{O}_{x_{\sigma}}\right)$-modules

$$
\left(L_{f}\right)_{\sigma}=\underset{\substack{\mu \geq f \text { on } \sigma \\ \mu \in M}}{\bigoplus} \boldsymbol{C} \cdot \mathfrak{X}^{\mu}
$$

can be patched together to an invertible sheaf $L_{f}$ on $\mathscr{X}_{\Sigma}$, and $L_{f}$ is ample if $\Sigma$ is a finite set. 


\section{§3. Compactification of Hilbert modular surfaces}

3.1. We review Hirzebruch's resolution of the cusps of a Hilbert modular surface (cf. [7]).

Let $N$ be a projective $\mathcal{O}$-module of rank $1, V$ a subgroup of finite index of $U_{+}$, the totally positive units of $\mathcal{O}$, and let $G(N, V)$ be the semidirect product

$$
\left\{\left(\begin{array}{cc}
\varepsilon & \mu \\
0 & 1
\end{array}\right) \in S L_{2}(K) \mid \varepsilon \in V, \mu \in N\right\}
$$

The module $N$ acts on $\mathscr{H}^{2}$ by translations

$$
\left(z_{1}, z_{2}\right) \longrightarrow\left(z_{1}+\mu^{(1)}, z_{2}+\mu^{(2)}\right), \quad \mu \in N .
$$

The quotient $\mathscr{H}^{2} / N$ can be considered as an open subset of the algebraic torus $C^{2} / N=\mathscr{T}$. We can identify the character group of $\mathscr{T}$ with the $Z$ dual $N^{*}$ of $N$ and the group of 1-parameter subgroups of $\mathscr{T}$ with $N$. This induces a pairing between $N$ and $N^{*}$ which coincides with that given by the trace.

We look for a rational partial polyhedral decomposition $\Sigma$ of $N_{R}=$ $N \otimes R$ which is invariant under the action of $V$, regular and such that the convex hull of $\Sigma$ coincides with the cone $N_{R}^{+}$of totally positive elements in $N_{R}$.

Consider the convex hull of $N_{R}^{+} \cap N$ in $N_{R}$. Its boundary points can be indexed by $Z$ such that if $A_{k}(k \in Z)$ are these points, $A_{k+1}>A_{k}$. Moreover, $A_{k}$ and $A_{k+1}$ form a basis of $N$ for each $k \in Z$.

The rational polyhedral decomposition $\Sigma$ of $N_{\boldsymbol{R}}^{+}$whose 1-simplices are spanned by the $A_{k}$ is a $V$-invariant regular decomposition of $N_{R}^{+}$.

The torus embedding $\mathscr{X}_{\Sigma}$ thus found contains a partial smooth compactification of $\mathscr{H}^{2} / N$. The exceptional divisor in it consists of an infinite chain of non-singular rational curves $C_{k}$ with $C_{k} \cdot C_{k \pm 1}=1, C_{k}^{2}=-b_{k}$ where $A_{k-1}+A_{k+1}=b_{k} A_{k}$ and $C_{k} \cdot C_{l}=0$ otherwise.

The action of a generator $\varepsilon$ of $V$ is properly discontinuous and sends $C_{k}$ to $C_{k+r}$ for some $r$. The exceptional divisor $\bigcup_{k} C_{k}$ projects down to a cycle of $r$ non-singular rational curves if $r \geq 2$ and to a rational curve with an ordinary double point if $r=1$. The quotient by $V$ forms a partial smooth compactification of $\mathscr{H}^{2} / G(N, V)$.

Since for each cusp $x$ of $\mathscr{H}^{2} / \Gamma, \Gamma$ a Hilbert modular group, there exists a neighbourhood of $x$ in $\mathscr{H}^{2} / \Gamma$ isomorphic to $W(d) / G(N, V)$, where $W(d)$ 
$=\left\{\left(z_{1}, z_{2}\right) \in \mathscr{H}^{2} \mid \operatorname{Im} z_{1} \cdot \operatorname{Im} z_{2}>d>0\right\}$, this procedure yields the resolution of all cusps of $\mathscr{H}^{2} / \Gamma$. Moreover, since all $b_{k} \geq 2$ it is the minimal resolution of these cusps.

3.2. The Hirzebruch decomposition described above is not projective with respect to the function

$$
\rho(y)=\min _{\substack{\nu \in N^{*} \\ \nu \gg 0}} \operatorname{Tr} \nu y
$$

on $N_{R}=N \otimes R$. Here $y=\left(y_{1}, y_{2}\right)$ are coordinates in $N_{R}$ derived from those in $\mathscr{H}^{2}$ : (i.e. $\left.\left(y_{1}, y_{2}\right)=\operatorname{ord}\left(z_{1}, z_{2}\right)\right)$ and $\operatorname{Tr} \nu y=\nu^{(1)} y_{1}+\nu^{(2)} y_{2}$. Since for each pair $A_{k-1}, A_{k}$ there exists a $V_{k} \in N_{+}^{*}$ such that $\operatorname{Tr} V_{k} A_{k-1}=\operatorname{Tr} V_{k} A_{k}=1, \rho$ is linear on each cone. Using this one easily verifies that $\rho$ satisfies the conditions i) and ii) of projectivity stated in (2.5). But $\rho$ satisfies iii) only if $b_{k}>2$ for all $k$, because $\rho$ is linear on the cone spanned by $A_{k-1}$ and $A_{k+1}$ if $A_{k-1}, A_{k}, A_{k+1}$ are collinear, i.e. $b_{k}=2$ (cf. [1], p. 305).

Removing the 1-simplices of the Hirzebruch decomposition spanned by those $A_{k}$ for which $b_{k}=2$ yields a new (but no longer regular) decomposition which we call the cuspidal decomposition. Its cones are the maximal cones on which $\rho$ is linear.

3.3. From the results of Baily-Borel ([2]) it follows that the cusp forms of sufficiently large weight with respect to a Hilbert modular group $\Gamma$ define an embedding of $\mathscr{H}^{2} / \Gamma$ as an open subset of a projective variety.

Let us suppose that $\Gamma$ acts freely on $\mathscr{H}^{2}$. Denote by $S_{m, n}$ the $C$-vector space of cusp forms for $\Gamma$ of weight $(m, n)$, i.e. satisfying

$$
f\left(\frac{a^{(1)} z_{1}+b^{(1)}}{c^{(1)} z_{1}+d^{(1)}}, \frac{a^{(2)} z_{2}+b^{(2)}}{c^{(2)} z_{2}+d^{(2)}}\right)=\left(c^{(1)} z_{1}+d^{(1)}\right)^{m}\left(c^{(2)} z_{2}+d^{(2)}\right)^{n}\left(f\left(z_{1}, z_{2}\right)\right) .
$$

If $S$ is the minimal smooth compactification of $\mathscr{H}^{2} / \Gamma$ and $K_{S}$ its canonical divisor class then $S_{2 m, 2 m}$ can be identified with $H^{0}\left(S, \mathcal{O}\left(m K_{S}+(m-1) D\right)\right.$ ), where $D$ is the divisor $\Sigma C_{k}$ consisting of all curves occurring in the cusp resolutions with multiplicity 1 .

Remark. The identification of $S_{2 m, 2 m}$ with $H^{0}\left(S, \mathcal{O}\left(m K_{S}+(m-1) D\right)\right)$ allows us to compute $\operatorname{dim} S_{2 m, 2 m}$. Assume for simplicity that $\Gamma$ operates freely. Then it follows from Hirzebruch's formulae that if

$$
D=\sum_{i=1}^{l} C_{i}, \quad C_{i}^{2}=-b_{i}
$$




$$
K_{S}^{2}=2 \operatorname{vol}\left(\mathscr{H}^{2} / \Gamma\right)+\sum_{i=1}^{l}\left(2-b_{i}\right)
$$

and

$$
K(K+D)=0
$$

From $\operatorname{dim} H^{1}\left(S, \mathcal{O}\left(m K_{S}+(m-1) D\right)=0\right.$ it follows by the Riemann-RochHirzebruch formula that

$$
\operatorname{dim} S_{2 m, 2 m}=\left(m^{2}-m\right) \operatorname{vol}\left(\mathscr{H}^{2} / \Gamma\right)+\chi\left(S, O_{S}\right) ;
$$

this is Shimizu's formula in our case. Note that we use the volume form

$$
-\frac{1}{16 \pi^{2}}\left(\operatorname{Im} z_{1} \operatorname{Im} z_{2}\right)^{-2} d z_{1} \wedge d \bar{z}_{1} \wedge d z_{2} \wedge d \bar{z}_{2} .
$$

\section{§4. A non-singular compactification of $\mathscr{A}^{0}$}

4.1. Let $\mathfrak{A}$ and $\mathfrak{B}$ be two projective $\mathcal{O}$-modules of rank 1 , and let $V$ be a group of units preserving $\mathfrak{A}$. We consider the algebraic torus $\mathscr{T}$

$$
C^{2} / \mathfrak{A} \times C^{2} / \mathfrak{B}
$$

which contains $\mathscr{H}^{2} / \mathfrak{A} \times C^{2} / \mathfrak{B}$ as an open subset. We assume $\mathfrak{A} \subset \mathfrak{B}$. The character group of $\mathscr{T}$ can be identified with $\mathfrak{B} \times \mathfrak{A}$. We look for a rational polyhedral decomposition $\Sigma$ of $\mathfrak{U}_{\boldsymbol{R}}^{+} \times \mathfrak{B}_{R}$ which satisfies

i) the projection of each cone in $\mathfrak{U}_{\boldsymbol{R}}^{+} \times \mathfrak{B}_{\boldsymbol{R}}$ onto $\mathfrak{U}_{\boldsymbol{R}}^{+}$is contained in a cone in $\mathfrak{U}_{R}^{+}$;

ii) the decomposition is invariant under

$$
\begin{array}{ll}
(x, y) \longrightarrow(\varepsilon x, \varepsilon y), & \varepsilon \in V, \\
(x, y) \longrightarrow(x, y+\alpha x), & \alpha \in \mathcal{O} ;
\end{array}
$$

iii) it is regular.

4.2. We begin by assuming that $\mathfrak{U}=\mathfrak{B}$. Let $\alpha \in \mathfrak{U}^{+}$. Consider the quadratic form on $\mathfrak{U}^{*}$ associated to $\alpha$ : $\operatorname{Tr} \xi^{2} \alpha\left(\xi \in \mathfrak{X}^{*}\right)$. The map

$$
\alpha \longrightarrow \operatorname{Tr} \xi^{2} \alpha
$$

extends $\boldsymbol{R}^{+}$-linearly to a map

$$
Q: \mathfrak{Y}_{R}^{+} \longrightarrow \mathscr{C}_{2},
$$

where $\mathscr{C}_{2}$ is the cone of positive semi-definite quadratic forms. Similarly, we may define $Q^{\beta}$ by associating to $\alpha \in \mathfrak{U}_{\boldsymbol{R}}^{+}$the form $\operatorname{Tr} \xi^{2} \alpha \beta$ for $\beta \in K$ with 
$\beta \mathfrak{A} \subset \mathcal{O}^{*}$ and $\beta \gg 0$. After choosing coordinates in $\mathscr{C}_{2}$ and a basis $\left\{u_{1}, u_{2}\right\}$ of $\mathfrak{U}^{*}$ we can write $Q^{\beta}$ as

$$
x \longrightarrow\left(\begin{array}{ll}
\operatorname{Tr} u_{1}^{2} \beta x & \operatorname{Tr} u_{1} u_{2} \beta x \\
\operatorname{Tr} u_{1} u_{2} \beta x & \operatorname{Tr} u_{2}^{2} \beta x
\end{array}\right) .
$$

Changing the basis of $\mathfrak{U}^{*}$ induces on $\mathscr{C}_{2}$ an action

$$
X \longrightarrow U X^{t} U, \quad X \in \mathscr{C}_{2},
$$

by $U \in G L(2, Z)$.

The coordinates $\eta_{i}$ of

$$
X=\left(\begin{array}{ll}
\eta_{1} & \eta_{2} \\
\eta_{2} & \eta_{3}
\end{array}\right)=Q^{\beta}(x)
$$

satisfy a linear relation of the form

$$
a \eta_{1}+b \eta_{2}+c \eta_{3}=0
$$

with integral coefficients. Equivalently, $X$ satisfies a relation

$$
E X-X^{t} E=0
$$

with $E \in M_{2}(Z)$. The number $\Delta=b^{2}-4 a c=(\operatorname{Tr} E)^{2}-4 \operatorname{det} E$ is invariant under the action of $G L(2, Z) \cap D^{-1} G L(2, Z) D$ and equals the discriminant of the $\mathcal{O}$-module $\mathfrak{A}^{*}$ :

$$
\operatorname{det}\left(\begin{array}{ll}
u_{1}^{(1)} & u_{2}^{(1)} \\
u_{1}^{(2)} & u_{2}^{(2)}
\end{array}\right)^{2}
$$

4.3. Let us assume that $\beta \mathfrak{U}=n \mathscr{O}^{*}, n \in N$.

A rational polyhedral decomposition of $\mathscr{C}_{2}$ which is invariant under $G L(2, Z)$ can be defined by equations

$$
E X-X^{t} E=0
$$

with invariant 1 for its 2-cells. The function

$$
\sum_{\alpha \in Z^{2} / 2 Z^{2}} \min _{\substack{\xi \in Z^{2} \\ \xi \equiv \alpha(\bmod 2)}} t \xi X \xi
$$

is linear on each cone and satisfies the conditions in 2.3. This decomposition coincides with the central cone decomposition of Igusa and Namikawa's Delaunay-Voronoi decomposition (cf. [13]). 
4.4. By means of $Q^{\beta}$ the decomposition of $\mathscr{C}_{2}$ induces a decomposition on $\mathfrak{U}_{R}^{+}$. In general it does not coincide with the Hirzebruch decomposition of $\mathfrak{X}_{R}^{+}$. Obviously, the function

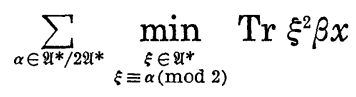

is linear on the cones of this decomposition.

4.5. Example. Let $p=89, K=\boldsymbol{Q}(\sqrt{p})$. We consider the map

$$
\left(z_{1}, z_{2}\right) \longrightarrow\left(\begin{array}{lll}
\operatorname{Tr} w^{2} \varepsilon_{0} \sqrt{p} z & \operatorname{Tr} w \varepsilon_{0} \sqrt{p} z \\
\operatorname{Tr} w \varepsilon_{0} \sqrt{p} z & \operatorname{Tr} & \varepsilon_{0} \sqrt{p} z
\end{array}\right)
$$

corresponding to a modular embedding of $S L_{2}(\mathcal{O})$ in $S p_{4}(Z)$. A comparison of the cone decompositions of $\mathcal{O}_{\boldsymbol{R}}^{+}$and $\mathscr{C}_{2}$ yields the following picture (here $\left.w=(1-\sqrt{89}) / 2, \varepsilon_{0}=500+53 \sqrt{89}\right)$. Here we represent $\mathscr{C}_{2}$ modulo scalars. On the image of $\mathscr{H}^{2}$ given by $\eta_{1}+\eta_{2}-22 \eta_{3}=0$ a number of consecutive rays of the Hirzebruch decomposition are marked.

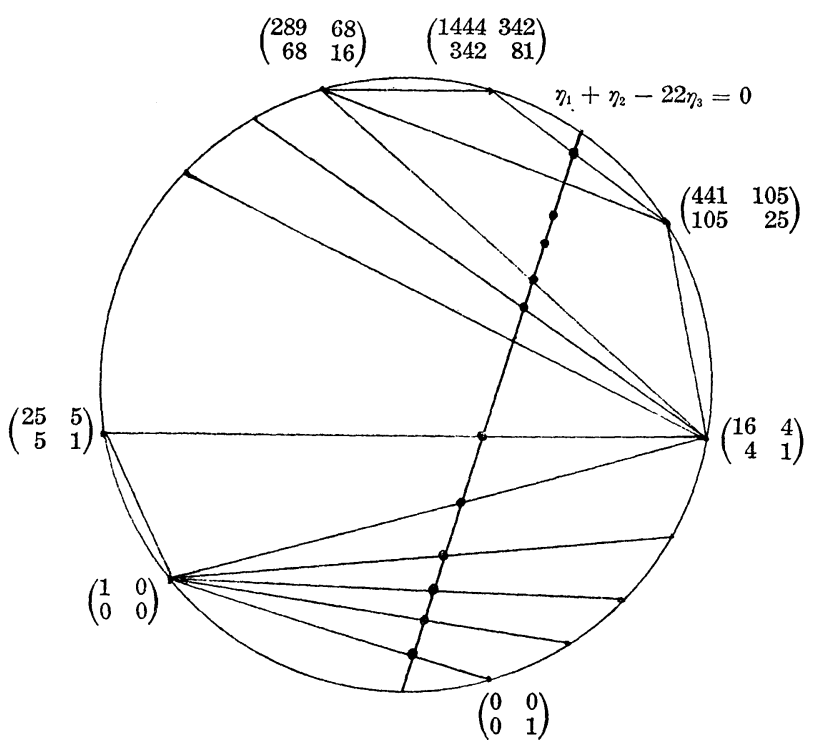

Fig. 1

4.6. Remark. Let $p=(2 a-1)^{2}+4$ be a prime and let

$$
\varepsilon_{0}=\frac{2 a-1+\sqrt{p}}{2}
$$

be the fundamental unit of $\boldsymbol{Q}(\sqrt{p})$. Consider the map $\mathcal{O}_{R}^{+} \rightarrow \mathscr{C}_{2}$ given by 


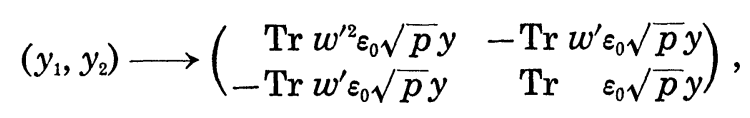

where $w=(1+\sqrt{p}) / 2$. Then the Hirzebruch decomposition coincides with the induced one.

4.7. Consider now the rational polyhedral decomposition $\Sigma_{1}$ of $\mathscr{C}_{2}$ whose 2-cells are defined by the equations $E X-X^{t} E=0\left(X \in \mathscr{C}_{2}\right)$ with invariant 1. Let $\sigma$ be the polyhedral cone spanned by

$$
\left(\begin{array}{ll}
1 & 0 \\
0 & 0
\end{array}\right), \quad\left(\begin{array}{ll}
0 & 0 \\
0 & 1
\end{array}\right) \text { and } \quad\left(\begin{array}{rr}
1 & -1 \\
-1 & 1
\end{array}\right)
$$

It is well-known that any polyhedral cone $\sigma^{\prime}$ in $\Sigma_{1}$ has a form $M \sigma^{t} M$ for certain $M \in G L(2, Z)$. Let $G(\sigma)$ be the subgroup of $G L(2, Z)$ which leaves $\sigma$ invariant. The group $G(\sigma)$ is a finite group and consists of twelve matrices.

$$
\begin{aligned}
& \pm\left(\begin{array}{ll}
1 & 0 \\
0 & 1
\end{array}\right), \pm\left(\begin{array}{rr}
1 & 0 \\
-1 & -1
\end{array}\right), \quad \pm\left(\begin{array}{rr}
1 & 1 \\
0 & -1
\end{array}\right) \\
& \pm\left(\begin{array}{ll}
0 & 1 \\
1 & 0
\end{array}\right), \quad \pm\left(\begin{array}{rr}
0 & -1 \\
1 & 1
\end{array}\right), \quad \pm\left(\begin{array}{rr}
-1 & -1 \\
1 & 0
\end{array}\right) .
\end{aligned}
$$

The group $G(\sigma)$ induces the automorphism group Aut $(\sigma)$ of $\sigma$ and Aut $(\sigma)$ is isomorphic to the symmetric group $S_{3}$ of degree 3. Let $M$ be one of the above twelve matrices and let us consider the action of $M$ on $R^{2}$ given by $y \mapsto y^{t} M$. Then it is easy to see that the triangulation of $R^{2}$ in Fig. 2 is invariant by this action where the vertices of triangles are the lattice points $Z^{2}$ in $R^{2}$.

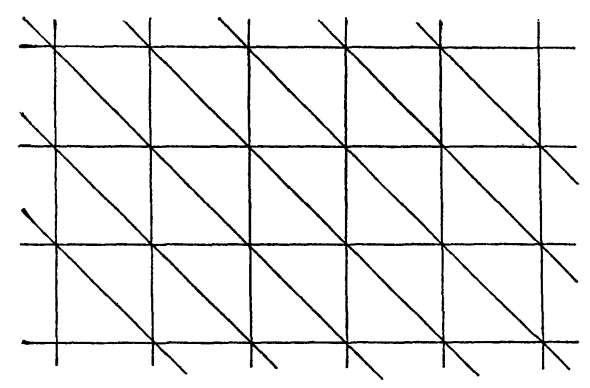

Fig. 2

Now using the map $Q^{\beta}$, from the polyhedral decomposition $\Sigma_{1}$ of $\mathscr{C}_{2}$, we obtain a decomposition of $\mathfrak{U}_{R}^{+}$. Let $\tilde{\Sigma}_{\beta}$ be the minimal refinement of this decomposition which is regular and assume that its 1-dimensional 
cones are spanned by $B_{k}, k \in Z$, with $B_{k}^{(1)}>B_{k-1}^{(1)}$. For a generator $\varepsilon$ of the unit group $V$ which preserve $\mathfrak{A}$, we may assume

$$
\varepsilon B_{0}=B_{l}, \quad l \geq 1 \text {. }
$$

Moreover we may assume that $B_{0}$ lies on a boundary of the polyhedral cone $\sigma$ spanned by

$$
\left(\begin{array}{ll}
1 & 0 \\
0 & 0
\end{array}\right),\left(\begin{array}{ll}
0 & 0 \\
0 & 1
\end{array}\right) \text { and }\left(\begin{array}{rr}
1 & -1 \\
-1 & 1
\end{array}\right)
$$

Let $\sigma_{1}, \sigma_{2}, \cdots, \sigma_{m}$ be polyhedral cones in $\Sigma_{1}$ which intersect the segment $Q^{\beta}\left(\overline{B_{0} B_{l}}\right)$ in $\mathscr{C}_{2}$. Let $B_{k_{1}}, B_{k_{2}}, \cdots, B_{k_{m}}, B_{l}$ be all the points which lie on the boundaries of these cones and $L_{i}$ the common boundary of $\sigma_{i}$ and $\sigma_{i+1}$, where we put $\sigma_{0}=\sigma$ and $\sigma_{m+1}$ is the polyhedral cone which contains the segment $Q^{\beta}\left(\overline{B_{l} B_{l+1}}\right)$. (see Fig. 3)

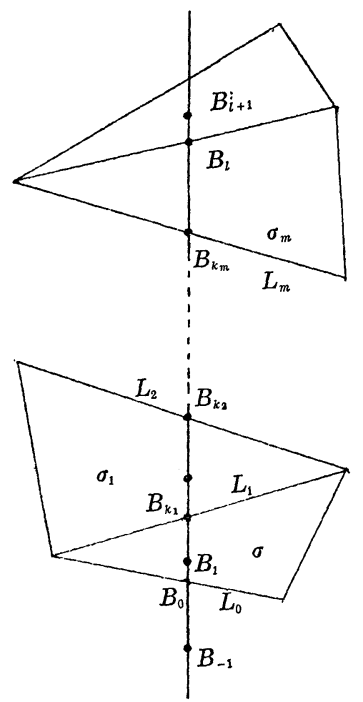

Fig. 3

We fix $M_{i} \in G L(2, Z), i=1,2, \cdots, m+1$ so that $M_{i} \sigma_{i}{ }^{t} M_{i}=\sigma_{i-1}$ and the action of $M_{i}$ on $L_{i}$ is trivial.

4.8. Now we construct a polyhedral decomposition of $\mathfrak{Y}_{R}^{+} \times \mathfrak{B}_{R}$. First assume that $Q^{\beta}\left(B_{i}\right)$ and $Q^{\beta}\left(B_{i+1}\right)$ lie in the cone $\sigma$. Then we can write

$$
Q^{\beta}\left(B_{j}\right)=\lambda_{j}\left(\begin{array}{ll}
1 & 0 \\
0 & 0
\end{array}\right)+\mu_{j}\left(\begin{array}{ll}
0 & 0 \\
0 & 1
\end{array}\right)+\nu_{j}\left(\begin{array}{rr}
1 & -1 \\
-1 & 1
\end{array}\right), \quad j=i, i+1
$$

with integers $\lambda_{i}, \lambda_{i+1}, \mu_{i}, \mu_{i+1}, \nu_{i}, \nu_{i+1}$. 
Put $x=s B_{i}+t B_{i+1}, s, t \geq 0$. We define a decomposition of $x \times \mathfrak{B}_{R}$ by giving it on a fundamental domain for the translations. We indicate an example in Fig. 4. Since it is obvious from this example how to treat the general case, we refrain from giving the equations for it.

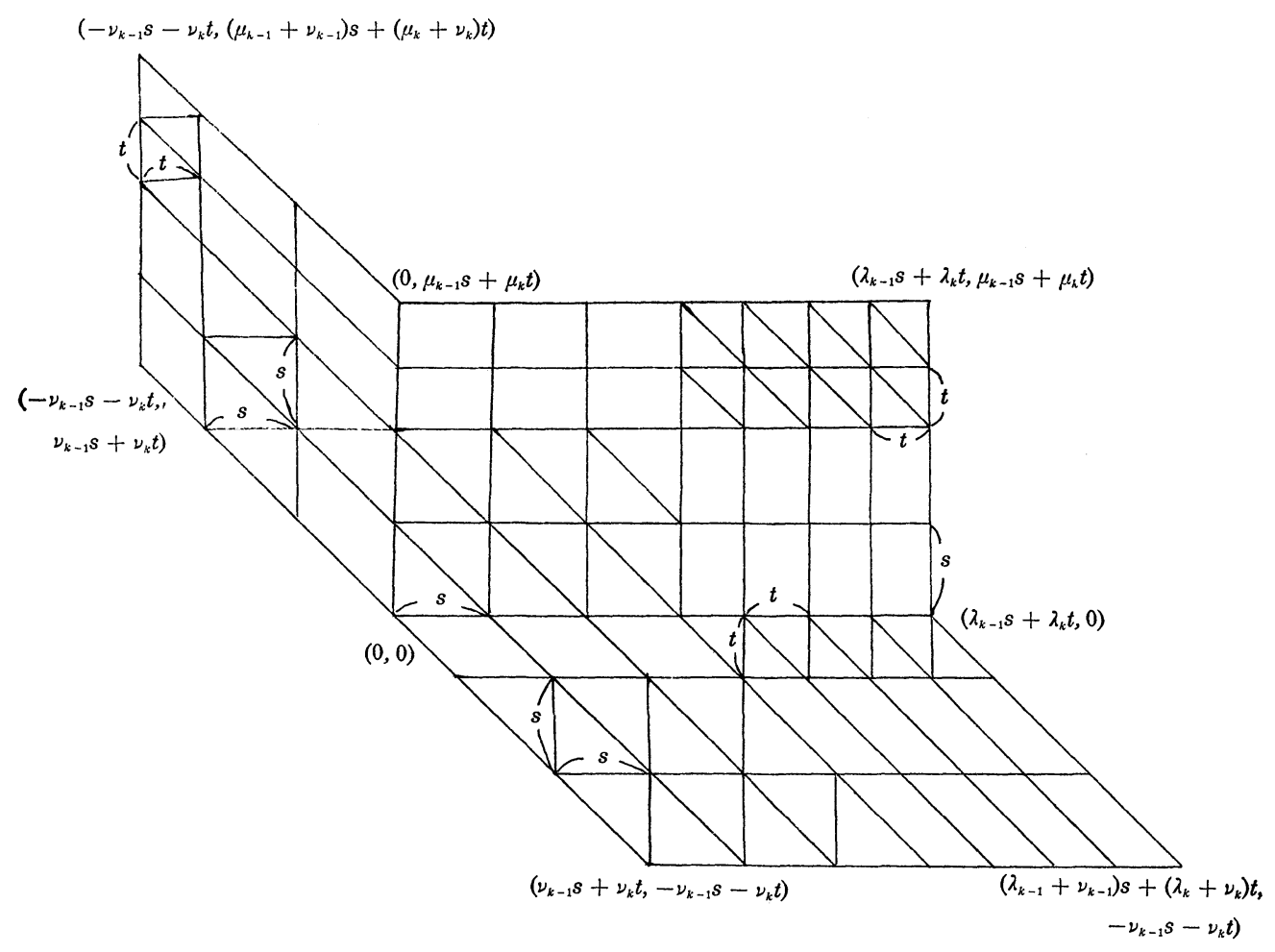

Fig. 4

(In this example $\lambda_{k-1}=3, \mu_{k-1}=2, \nu_{k-1}=2, \lambda_{k}=4, \mu_{k}=2, \nu_{k}=1$ ).

Next if $Q^{\beta}\left(B_{i}\right)$ and $Q^{\beta}\left(B_{i+1}\right)$ lie in $\sigma_{1}$, then by the choice of $M_{1} \in$ $G L(2, Z)$ in 4.7, $M_{1} Q^{\beta}\left(B_{i}\right)^{t} M_{1}$ and $M_{1} Q^{\beta}\left(B_{i+1}\right)^{t} M_{1}$ lie in $\sigma$.

For $x=s B_{i}+t B_{i+1}$ we have a decomposition of $x \times B_{R}$ by means of $M_{1} Q^{\beta}\left(B_{i}\right)^{t} M_{1}$ and $M_{1} Q^{\beta}\left(B_{i+1}\right)^{t} M_{1}$. We then let $M_{1}^{-1}$ act on $\mathfrak{B}_{R}: y \mapsto y^{t} M^{-1}$ and obtain the required decomposition of $x \times \mathfrak{B}_{R}$. Since $M_{1}$ acts on $L_{1}$ trivially, the induced decompositions of $B_{k_{1}} \times \mathfrak{B}_{R}$ given by $\tau_{k_{1-1}} \times \mathfrak{B}_{\boldsymbol{R}}$ and $\tau_{k_{1}} \times \mathfrak{B}_{\boldsymbol{R}}$ coincide, where $\tau_{j}$ is the cone of $\mathfrak{2}_{R}^{+}$spanned by $B_{j}$ and $B_{j+1}$. Next if $Q^{\beta}\left(B_{i}\right)$ and $Q^{\beta}\left(B_{i+1}\right)$ lie in $\sigma_{2}$, then by the choice of $M_{2} \in G L(2, Z)$ in 4.7, $M_{2} Q^{\beta}\left(B_{i}\right)^{t} M_{2}$ and $M_{2} Q^{\beta}\left(B_{i+1}\right)^{t} M_{2}$ lie in $\sigma_{1}$. Hence we can apply the above method. In this way, by induction we obtain a polyhedral cone decom- 
positions of $\tau_{j} \times \mathfrak{B}_{R}$, where $\tau_{j}$ is the polyhedral cone of $\mathfrak{A}_{R}^{+}$spanned by $B_{j}$ and $B_{j+1}$, and we can glue together the decompositions of $\tau_{k} \times \mathfrak{B}_{R}$ and $\tau_{k+1} \times \mathfrak{B}_{R}$. We repeat the process until $k=l-1$. Then, using the action of the unit group $V$, we obtain a decomposition of $\mathfrak{A}_{R}^{+} \times \mathfrak{B}_{R}$. We need to check that this decomposition is well-defined. For that purpose, it is enough to show that the induced decomposition $\varepsilon \cdot\left(B_{0} \times \mathfrak{B}_{R}\right)$ by the action of the unit $\varepsilon$ and the decomposition $B_{l} \times \mathfrak{B}_{R}$ coincide. (Note that $B_{l}=$ $\varepsilon \cdot B_{0}$, See 4.7.) From the definition of $M_{i}, i=1,2, \cdots, m+1$ and the decomposition of $x \times \mathfrak{B}_{R}$, the decomposition of $B_{l} \times \mathfrak{B}_{R}$, is obtained by the decomposition of $\boldsymbol{R}^{2}$ given in Fig. 4 by means of $M=M_{1} M_{2} \cdots M_{m+1}$. (Note that $M_{m+1}$ acts trivially on $L_{m+1}$ ). On the other hand by the modular imbedding, the unit $\varepsilon$ defines an element $\phi(\varepsilon) \in G L(2, Z)$ and the action of $\varepsilon$ on $\mathscr{C}_{2}$ is given by $X \mapsto \phi(\varepsilon) X^{t} \phi(\varepsilon)$. As $M \sigma_{m+1}^{t} M=\sigma$ and $\phi(\varepsilon) \sigma^{t} \phi(\varepsilon)=\sigma_{m+1}$, we have $M^{-1} \phi(\varepsilon) \in G(\sigma)$. Since the decomposition of $R^{2}$ given in Fig. 2 is invariant by the action of $M^{-1} \phi(\varepsilon)$, the decomposition $\varepsilon \cdot\left(B_{0} \times \mathfrak{B}_{R}\right)$ and $B_{l}$ $\times \mathfrak{B}_{R}$ coincide. Thus we obtain a polyhedral decomposition of $\mathfrak{N}_{R}^{+} \times \mathfrak{B}_{R}$ which satisfies i), ii) and iii) in 4.1 .

4.9. In paragraphs $4.3-4.8$ we constructed a decomposition of $\mathfrak{Y}_{\boldsymbol{R}}^{+} \times$ $\mathfrak{B}_{R}$ under the assumption that $\beta \mathfrak{A}=n \mathcal{O}^{*}$ and $\mathfrak{B}=\mathcal{O}$.

First note that by changing $\mathfrak{A}$, if necessary, we may assume $\beta=1$. So in the general case we have $\mathfrak{A} \subset \mathfrak{B} \subset \mathcal{O}^{*}$ and we then proceed as follows.

For $x \in \mathfrak{Y}_{\boldsymbol{R}}^{+}$let $\operatorname{Tr} \xi^{2} x$ be the associated quadratic form on $\mathfrak{B}^{*}$. The metric on $\mathfrak{B}_{R}^{*}$ induced by this quadratic form can be used to define polyhedral decompositions of $\mathfrak{B}_{R}^{*}$ and $\mathfrak{B}_{\boldsymbol{R}}$ (cf. [11] and [13]). We say that a set of elements of $\mathfrak{B}^{*}\left\{a_{i}\right\}_{i \in I}$ spans a Delaunay cell $D\left(a_{i}\right)$ with respect to $x$ if there exists a $\gamma \in \mathfrak{B}_{R}^{*}$ such that

$$
\left\{a_{i}\right\}_{i \in I}=\left\{\eta \in \mathfrak{B}^{*} \mid d_{x}(\gamma, \eta)=\min _{\xi \in \mathfrak{B}^{*}} d_{x}(\gamma, \xi)\right\} .
$$

These cells define a polyhedral decomposition of $\mathfrak{B}_{\boldsymbol{R}}^{*}$ by bounded cells, each of which has a finite number of faces. This is the Delaunay decomposition of $\mathfrak{B}_{\boldsymbol{R}}^{*}$.

The 'dual' decomposition of $\mathfrak{B}_{\boldsymbol{R}}$ is obtained by associating to each Delaunay cell $D\left(a_{i}\right)$ the Voronoi cell

$$
\Delta\left(a_{i}\right)=\left\{\begin{array}{c}
y \in \mathfrak{B}_{R} \mid \operatorname{Tr}\left(\left(\xi+a_{i}\right)^{2} x+\left(\xi+a_{i}\right) y\right) \geq \operatorname{Tr}\left(a_{i}^{2} x+a_{i} y\right) \\
\forall \xi \in \mathfrak{B}^{*}, \forall i \in I
\end{array}\right\}
$$


This decomposition, which is invariant under translations

$$
y \longrightarrow y+2 \xi x \quad\left(\xi \in \mathfrak{B}^{*}\right),
$$

is called the Voronoi decomposition.

Define an equivalence relation on $\mathfrak{U}_{R}^{+}$by $x \sim x^{\prime}$ if the Delaunay decompositions of $\mathfrak{B}_{R}^{*}$ defined by $x$ and $x^{\prime}$ coincide. The closure of an equivalence class in $\mathfrak{U}_{R}^{+}$is an unbounded polyhedral cone in $\mathfrak{U}_{R}^{+}$. We obtain a polyhedral decomposition $\Sigma$ of $\mathfrak{U}_{\boldsymbol{R}}^{+}$. The equivalence

$$
\operatorname{Tr}\left(\xi\left(\xi+2 a_{i}\right) x+\xi y\right) \geq 0 \Longleftrightarrow \operatorname{Tr}\left(\xi\left(\xi+2 a_{i}+\rho\right) x+\xi(y-\rho x)\right) \geq 0
$$

implies that the Voronoi decomposition associated to

$$
\operatorname{Tr}\left((\xi+\alpha)^{2} x+(\xi+\alpha) y\right) \text { with } \alpha \in \frac{1}{2} \mathfrak{B}^{*} / \mathfrak{B}^{*}
$$

is the translation over a half period of the Voronoi decomposition associated to $\operatorname{Tr}\left(\xi^{2} x+\xi y\right)$. The superposition of the Voronoi decompositions associated to $\operatorname{Tr}\left((\xi+\alpha)^{2} x+(\xi+\alpha) y\right)$ for all $\alpha \in \frac{1}{2} \mathfrak{B}^{*} / \mathfrak{B}^{*}$ is a subdivision of the Voronoi decomposition (see Fig. 5).

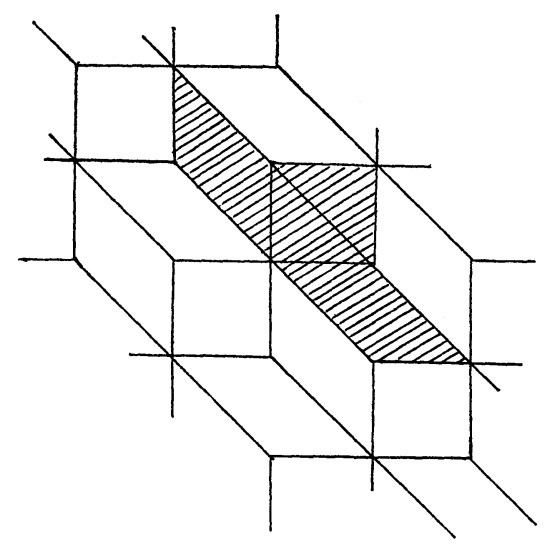

Fig. 5

4.10. Now we define several decompositions of $\mathfrak{P}_{R}^{+} \times \mathfrak{B}_{R}$. The mixed decomposition is the decomposition of $\mathfrak{U}_{R}^{+} \times \mathfrak{B}_{R}$ which induces $\Sigma$ on $\mathfrak{Y}_{R}^{+}$ and on $x \times \mathfrak{B}_{R}$ the Voronoi decomposition defined by $x \in \mathfrak{Y}_{R}^{+}$.

The half period decomposition is the subdivision of the mixed decomposition obtained by translating in each fibre $x \times \mathfrak{B}_{R}$ the Voronoi decomposition over the half periods. It is the maximal decomposition of $\mathfrak{U}_{\boldsymbol{R}}^{+} \times$ $\mathfrak{B}_{R}$ on whose cones the four functions 


$$
f_{\rho}=\min _{\xi \in \mathfrak{B}^{*}} \operatorname{Tr}\left((\xi+\rho)^{2} x+(\xi+\rho) y\right) \quad \rho \in \frac{1}{2} \mathfrak{B}^{*} / \mathfrak{B}^{*}
$$

are linear. This decomposition is not regular but satisfies conditions i) and ii) of 4.1. We now show how to make it regular. We start by constructing in a minimal way a refinement $\tilde{\Sigma}$ of $\Sigma$ which is regular and such that the image of every 2-dimensional cone of $\tilde{\Sigma}$ under $Q^{\beta}$ is contained in a fundamental domain for the action of the image of the unit group $V$ in $G L(2, Z)$.

Suppose that $A_{1}, A_{2} \in \mathfrak{Q}^{+}$span a 2-dimensional cone of $\tilde{\Sigma}$. Then $A_{1}$ and $A_{2}$ belong to the same equivalence class in $\mathfrak{U}_{R}^{+}$. Consequently, there exist elements $b_{1}, b_{2} \in \mathfrak{B}$ such that the Voronoi cell containing $(0,0)$ defined by $A_{i}(i=1,2)$ is the convex hull of the points

$$
\left\{ \pm \lambda_{i} b_{1}, \pm \mu_{i} b_{2}, \pm \nu_{i}\left(b_{1}+b_{2}\right)\right\} \quad\left(\lambda_{i}, \mu_{i}, \nu_{i} \in Z\right) .
$$

So the Voronoi cell containing $(0,0) \in \mathfrak{B}_{R}$ defined by $x=s A_{1}+t A_{2}$ $(s, t \in R)$ is the convex hull of the points

$$
\left\{ \pm\left(s \lambda_{1}+t \lambda_{2}\right) b_{1}, \pm\left(s \mu_{1}+t \mu_{2}\right) b_{2}, \pm\left(s \nu_{1}+t \nu_{2}\right)\left(b_{1}+b_{2}\right)\right\}
$$

Translating the Voronoi decomposition over the half periods yields a subdivision as in Fig. 5. Choose a fundamental domain $\mathscr{D}$ in $\mathscr{C}_{2}$ for the action of the group $V$ consisting of transforms under $G L(2, Z)$ of a fixed cone of $\Sigma_{4}$. Fix for the cones of $\tilde{\Sigma}$ whose image under $Q^{\beta}$ lies in $\mathscr{D}$ a choice of $b_{1}$ and $b_{2}$. Having fixed $b_{1}$ and $b_{2}$ we can apply the division process explained in 4.8 (cf. Fig. 3) to the shaded domain in Fig. 5 and by covering $\mathfrak{B}_{R}$ by its translates. We get a polyhedral decomposition of $\mathfrak{B}_{R}$, which we call a regular Voronoi decomposition w.r.t. $x=s A_{1}$ $+t A_{2}$. Note that it depends on a choice of $b_{1}$ and $b_{2}$ for the cones of $\tilde{\Sigma}$ contained in this fundamental domain. However, in the following we shall assume that we have made such a choice and simply speak of the regular Voronoi decomposition w.r.t. $x$. By an inductive process as in 4.8 we now can construct a decomposition of $\mathfrak{U}_{\boldsymbol{R}}^{+} \times \mathfrak{B}_{\boldsymbol{R}}$ which satisfies the requirements of 4.1. We call it the regular half period decomposition. Note that this decomposition is different from the one constructed in 4.7-4.8.

4.11. To the mixed decomposition (resp. the half period decomposition, resp. the regular half period decomposition) we associate a torus embedding containing $\mathscr{H}^{2} / \mathfrak{U} \times C^{2} / \mathfrak{B}$ which we denote by $\mathscr{X}_{1}(\mathfrak{U}, \mathfrak{B})\left(\operatorname{resp} . \mathscr{X}_{\frac{1}{2}}(\mathfrak{A}, \mathfrak{B})\right.$, 
$\left.\widetilde{\mathscr{X}}_{\frac{1}{2}}(\mathfrak{H}, \mathfrak{B})\right)$. It is a scheme which is locally of finite type. We refer to [13] for a description of the fibres of $\mathscr{X}_{1}(\mathfrak{Y}, \mathfrak{B}) \rightarrow \mathscr{Y}(\mathfrak{V})$, where $\mathscr{Y}(\mathfrak{V})$ is the 2-dimensional torus embedding defined by $\Sigma$. The fibres of $\mathscr{X}_{\frac{1}{2}}(\mathfrak{A}, \mathfrak{B}) \rightarrow$ $\mathscr{Y}(\mathfrak{C}) \quad\left(\right.$ resp. $\quad \widetilde{\mathscr{X}}_{\frac{1}{2}}(\mathfrak{X}, \mathfrak{B}) \rightarrow \widetilde{\mathscr{Y}}(\mathfrak{U})$, where $\tilde{\mathscr{Y}}(\mathfrak{R})$ corresponds to $\tilde{\Sigma}$ ) over limit points $\lim _{t \rightarrow 0} t^{\alpha}\left(\alpha \in \mathfrak{A}_{R}^{+}\right)$in $\mathscr{Y}(\mathfrak{U})$ (resp. $\widetilde{\mathscr{Y}}(\mathfrak{U})$ ) are infinite unions of rational surfaces. The dual graph of such a fibre is given by the decomposition of $\alpha \times \mathfrak{B}_{R}$.

If $\varepsilon$ is a totally positive unit preserving $\mathfrak{U}$ and $\mathfrak{B}$, then the action of $\varepsilon$ on $\mathscr{H}^{2} \times C^{2}$ extends to a well-defined action of $\varepsilon$ on $\mathscr{X}_{1}(\mathscr{X}, \mathfrak{B})$ and $\mathscr{Y}(\mathfrak{U})$ (resp. $\mathscr{X}_{\frac{1}{2}}(\mathfrak{U}, \mathfrak{B})$ and $\mathscr{Y}(\mathfrak{U}), \widetilde{\mathscr{X}}_{\frac{1}{2}}(\mathfrak{Y}, \mathfrak{B})$ and $\widetilde{\mathscr{Y}}(\mathfrak{Y})$ ) which is equivariant with respect to the projection $\mathscr{X}_{1}(\mathfrak{X}, \mathfrak{B}) \rightarrow \mathscr{Y}(\mathfrak{X}) \quad\left(\right.$ resp. $\mathscr{X}_{\frac{1}{2}}(\mathfrak{H}, \mathfrak{B}) \rightarrow \mathscr{Y}(\mathfrak{X})$, $\left.\widetilde{\mathscr{X}}_{\frac{1}{2}}(\mathfrak{Y}, \mathfrak{B}) \rightarrow \widetilde{\mathscr{Y}}(\mathfrak{Q})\right)$ and discontinuous on the closure of $W(d) \times C^{2}$,

4.12. Let $\Gamma$ be a principal congruence group of $S L_{2}\left(\mathcal{O}, \mathfrak{B}^{\prime}\right)$ which acts freely on $\mathscr{H}^{2}$, where $\mathfrak{B}^{\prime}$ is a projective $\mathcal{O}$-module of rank 1 . Consider the action of $\Gamma$ on $\mathscr{H}^{2} \times C^{2}$ as defined in 1.10. Let $s$ denote a cusp and let $\rho \in S L_{2}(K)$ with $\rho s=\infty$. The stabilizer $\Gamma_{\infty}$ of $\infty$ in $\rho \Gamma \rho^{-1}$ is the semidirect product $G(V, \mathfrak{U})$ of an $\mathcal{O}$-modulo $\mathfrak{A}$ and a group $V$ of units preserving $\mathfrak{A}$. Let $\rho \Gamma \rho^{-1}=S L_{2}\left(\mathcal{O}, \mathfrak{B}^{\prime} \mathfrak{S}^{2}\right)$ for some ideal $\mathfrak{E}$ and set $\mathfrak{B}=\mathfrak{B}^{\prime} \mathfrak{S}^{2}$. Then $\mathfrak{A} \subset \mathfrak{B}$ and we may assume $\mathfrak{B} \subset \mathcal{O}^{*}$. There exists a $\Gamma_{\infty}$-invariant neighbourhood $W=W(d)=\left\{\left(z_{1}, z_{2}\right) \in \mathscr{H}^{2} \mid \operatorname{Im} z_{1} \cdot \operatorname{Im} z_{2}>d\right\}$ of $\infty$ such that whenever $(z, \zeta),\left(z^{\prime}, \zeta^{\prime}\right) \in W \times C^{2}$ and $\gamma \in \rho \Gamma \rho^{-1}$ the equation $\gamma(z, \zeta)=\left(z^{\prime}, \zeta^{\prime}\right)$ implies $\gamma \in \Gamma_{\infty}$. The product $\mathfrak{U} \times \mathfrak{B}$ acts on $W \times C^{2}$ and we have the inclusions

$$
W \times C^{2} / \mathfrak{A} \times \mathfrak{B} \subset \mathscr{H}^{2} \times C^{2} / \mathfrak{U} \times \mathfrak{B} \subset C^{2} / \mathfrak{A} \times C^{2} / \mathfrak{B} .
$$

Hence by the theory of torus embeddings each of the rational polyhedral decompositions of $\mathfrak{U}_{R}^{+} \times \mathfrak{B}_{R}$ considered in 4.10 yields an embedding

$$
W \times C^{2} / \mathfrak{U} \times \mathfrak{B} \subset \mathscr{X}(\mathfrak{A}, \mathfrak{B}) \quad\left(\mathscr{X}=\mathscr{X}_{1}, \mathscr{X}_{\frac{1}{2}} \text { or } \widetilde{\mathscr{X}}_{\frac{1}{2}}\right)
$$

which is equivariant with respect to the action of $V$. By gluing $\mathscr{H}^{2} \times$ $C^{2} / \Gamma \times \mathfrak{B}^{\prime}$ with $\mathscr{X}(\mathfrak{A}, \mathfrak{B})$ over $W \times C^{2} / G(V, \mathfrak{X}) \times \mathfrak{B}$ and carrying out this construction for all cusps we obtain a fibre space $\mathscr{X}(\Gamma, \mathscr{M})\left(\mathscr{M}=\mathcal{O} \oplus \mathfrak{B}^{\prime}\right.$, $\mathscr{X}=\mathscr{X}_{1}, \mathscr{X}_{\frac{1}{2}}, \widetilde{\mathscr{X}}_{\frac{1}{2}}$ ) over a compactified Hilbert modular surface (which we denote by $S(\Gamma)$ if $\mathscr{X}=\mathscr{X}_{1}$ or $\mathscr{X}_{\frac{1}{2}}$ and by $\tilde{S}(\Gamma)$ if $\mathscr{X}=\widetilde{\mathscr{X}}_{\frac{1}{2}}$ ).

Following Namikawa we call each of these fibre spaces a family of semi-universal coverings.

Next we define an action of $\mathcal{O}$ on the fibres of $\mathscr{X}(\Gamma, \mathscr{M})$. The action of $\alpha \in \mathcal{O}$ on $\mathscr{H}^{2} \times C^{2}$ 


$$
\left(z_{1}, z_{2} ; \zeta_{1}, \zeta_{2}\right) \longrightarrow\left(z_{1}, z_{2} ; \zeta_{1}+\alpha^{(1)} z_{1}, \zeta_{2}+\alpha^{(2)} z_{2}\right)
$$

is compatible with the action of $\Gamma \ltimes \mathfrak{A}$ on $\mathscr{H}^{2} \times C^{2}$ and induces an action of $\alpha$ on $\mathscr{H}^{2} \times C^{2} / \Gamma \ltimes \mathfrak{B}^{\prime}$ and thus on $W \times C^{2} / \mathfrak{A} \ltimes \mathfrak{B}$. It extends to an action on $\mathscr{X}_{\frac{1}{2}}(\mathfrak{A}, \mathfrak{B})$ and on $\widetilde{\mathscr{X}}_{\frac{1}{2}}(\mathfrak{Y}, \mathfrak{B})$, which is equivariant with respect to the action of $V$. In this way we obtain an action of $\mathcal{O}$ on $\mathscr{X}_{\frac{1}{2}}(\Gamma, \mathscr{M})$ and on $\mathscr{X}_{\frac{1}{2}}(\Gamma, \mathscr{M})$ and one easily shows that it is properly discontinuous and fixed point free. By taking the quotient we obtain families

$$
\pi: \mathscr{A}_{\frac{1}{2}}(\Gamma, \mathscr{M}) \longrightarrow S(\Gamma)
$$

and

$$
\tilde{\pi}: \tilde{\mathscr{A}}_{\frac{1}{1}}(\Gamma, \mathscr{M}) \longrightarrow \tilde{S}(\Gamma)
$$

of so-called stable quasi-abelian surfaces. Note that $\tilde{\mathscr{A}}_{\frac{1}{2}}(\Gamma, \mathscr{M})$ and $\tilde{S}(\Gamma)$ are non-singular.

In case $\mathscr{X}=\mathscr{X}_{1}$ we proceed differently. We assume that $\Gamma$ is a principal congruence subgroup of $S L_{2}\left(\mathcal{O}, \mathfrak{B}^{\prime}\right)$ of level $\mathfrak{A} \subset \mathfrak{B}$ with (2)|\{r. Consider the surjection

$$
\mathscr{H}^{2} \times C^{2} \stackrel{\Psi}{\longrightarrow} \mathscr{H}^{2} \times C^{2} \longrightarrow \mathscr{H}^{2} / \mathfrak{U} \times C^{2} / \mathfrak{B},
$$

where $\Psi(z ; \zeta)=(z / 2, \zeta)$. The action of $\mathcal{O}$ on $\mathscr{H}^{2} \times C^{2}$ defines an action of $\mathcal{O}$ on the torus embedding $\mathscr{X}_{1}(\mathfrak{H}, \mathfrak{B})$ which contains $\mathscr{H}^{2} / \mathfrak{U} \times C^{2} / \mathfrak{B}$. It is properly discontinuous and fixpoint free.

By taking the quotient we obtain another family

$$
\pi^{(2)}:{ }^{(2)} \mathscr{A}_{1}(\Gamma, \mathscr{M}) \longrightarrow S(\Gamma)
$$

of stable quasi-abelian surfaces.

\section{§. Simple connectedness of $\mathscr{A}$}

5.1. Theorem. The analytic fibre spaces of stable quasi-abelian surfaces $\mathscr{A}=\mathscr{A}_{\frac{1}{3}}(\Gamma, \mathscr{M}), \tilde{\mathscr{A}}_{\frac{1}{2}}(\Gamma, \mathscr{M})$ and ${ }^{(2)} \mathscr{A}_{1}(\Gamma, \mathscr{M})$ (with $\mathscr{M}=\mathscr{O} \oplus \mathfrak{B}^{\prime}, \Gamma \subset$ $\left.S L_{2}\left(\mathcal{O}, \mathfrak{B}^{\prime}\right)\right)$ are simply-connected.

Proof. Let $U_{1}, \cdots, U_{n}$ be (suitable) neighbourhoods of the connected components of $\mathscr{A}-\mathscr{A}^{0}$. Choose base points $p_{i} \in \mathscr{A}-\mathscr{A}^{0}, p_{i} \in U_{i}$. Denote by $K_{i}$ the kernel of $\pi_{1}\left(U_{1} \cap \mathscr{A}^{0}, p_{i}\right) \rightarrow \pi_{1}\left(U_{i}, p_{i}\right)$. By Van Kampen's theorem $\pi_{1}(\mathscr{A})=\pi_{1}\left(\mathscr{A}^{0}, p\right) / N$, where $N$ is the smallest normal subgroup of $\Gamma \ltimes \mathscr{M}$ 
which contains $K_{i}, i=1, \cdots, n$. Now let $s$ be a cusp and let $\rho \in S L_{2}(K)$ with $\rho s=\infty$. Set $\Gamma_{\infty}=\rho \Gamma_{s} \rho^{-1}$, and let $T_{s}$ be the translation subgroup of $\Gamma_{\infty}$. Then $\rho \Gamma_{s} \rho^{-1}=G(V, \mathfrak{X})$ for some $\mathfrak{U}$ (cf. 3.1 ) and we construct a torus embedding $\mathscr{X}(\mathfrak{A}, \mathfrak{B})$ as in 4.9. Let $U$ be the interior of the closure of $W(d) / \mathfrak{X} \times C^{2} / \mathfrak{B}$ in $\mathscr{X}(\mathfrak{A}, \mathfrak{B})$, where $W(d)=\left\{\left(z_{1}, z_{2}\right) \in \mathscr{H}^{2} \mid \operatorname{Im} z_{1} \operatorname{Im} z_{2}>d\right)$ for sufficiently large $d$. Then $U$ is simply-connected. Hence $N$ contains $\rho^{-1}\left(T_{s} \times \mathfrak{B}\right) \rho$, in particular $N$ contains $\rho^{-1} T_{s} \rho$ for all $s$. But then, by a theorem of Vaserstein (cf. [16]), $N$ contains $\Gamma$. By specializing $s=\infty$ (resp. $s=0$ ) we see that $N$ contains

$$
\begin{aligned}
& \left\{\left(\left(\begin{array}{ll}
1 & x \\
0 & 1
\end{array}\right),\left(\begin{array}{ll}
0 & \beta
\end{array}\right)\right) \in \Gamma \ltimes \mathscr{M} \mid x \in \mathfrak{B}, \beta \in \mathfrak{B}^{*}\right\} \\
& \left(\operatorname{resp.}\left\{\left(\left(\begin{array}{ll}
1 & 0 \\
y & 1
\end{array}\right),\left(\begin{array}{ll}
\alpha & 0))
\end{array}\right) \in \Gamma \ltimes \mathscr{M} \mid y \in \mathfrak{B}^{-1}, \alpha \in \mathcal{O}\right\}\right) .\right.
\end{aligned}
$$

Consequently, $N$ contains $\Gamma \ltimes \mathscr{M}$.

\section{§6. Holomorphic forms on $\mathscr{A}$}

In this section we consider the relation between holomorphic forms on $\mathscr{A}$ and Hilbert modular forms.

6.1. Let us consider a modular embedding of a Hilbert modular group $S L_{2}(\mathcal{O})$ where $\mathcal{O}$ is the integer ring of a totally real number field of degree $n$. For a subgroup $\Gamma \subset S L_{2}(\mathcal{O})$ which operates properly discontinuously and freely on $\mathscr{H}^{n}$, we have an action of $G=\Gamma \ltimes\left(Z^{2 n} \times \cdots \times Z^{2 n}\right)$ on $\mathscr{H}^{n} \times C^{2 m}$ such that the quotient manifold $\mathscr{A}^{0}(m)=\mathscr{H}^{n} \times C^{2 m} / G$ is a smooth family of $m$-fold products of $n$-dimensional polarized abelian varieties over $S^{0}=\mathscr{H}^{n} / \Gamma$. By $S_{k_{1}, \cdots, k_{n}}$ we denote the space of Hilbert modular cusp forms of type $\left(k_{1}, \cdots, k_{n}\right)$. Suppose that $k_{i} \geq 2$. Then, to each $f \in S_{k_{1}, \cdots, k_{n}}$ and subsets $N_{j}, j=1,2, \cdots, m$, of $\{1, \cdots, n\}$ satisfying $k_{i}$ $-2=\sharp\left\{N_{l} \mid i \in N_{l}\right\}$, we can associate a g-invariant holomorphic $d$-form $\tilde{\omega}_{f}$ on $\mathscr{H}^{n} \times \boldsymbol{C}^{n m}$ defined by

$$
\tilde{\omega}_{f}=f\left(z_{1}, \cdots, z_{n}\right) d z_{1} \wedge \cdots \wedge d z_{n} \wedge d \zeta_{N_{1}}^{(1)} \wedge \cdots \wedge d \zeta_{N_{m}}^{(m)}
$$

where

$$
\begin{aligned}
& d=n+\sum_{i=1}^{m} N_{i} \\
& d \zeta_{N_{i}}^{(i)}=d \zeta_{k_{1}}^{(i)} \wedge \cdots \wedge d \zeta_{k_{l}}^{(i)}, \quad N_{i}=\left\{k_{1}, \cdots, k_{l}\right\}, \quad k_{1}<\cdots<k_{l} .
\end{aligned}
$$


Then $\tilde{\omega}_{f}$ is $G$-invariant, hence defines a holomorphic $d$-form $\omega_{f}$ on $\mathscr{A}^{0}(m)$.

Note that any Hilbert modular form of type $\left(k_{1}, \cdots, k_{n}\right)$ is a cusp form, if $k_{1}, \cdots, k_{n}$ are not the same.

Proposition 1. The form $\omega_{f}$ can be extended to a holomorphic $d$ form on $\mathscr{A}(m)$, where $\pi: \mathscr{A}(m) \rightarrow S$ is any non-singular compactification of $\pi^{0}: \mathscr{A}^{0}(m) \rightarrow S^{0}$.

Proof. It is enough to show that $\omega_{f}$ can be extended to a holomorphic form on a non-singular compactification $\mathscr{A}(m)$. Therefore, we may assume that $\pi: \mathscr{A}(m) \rightarrow S$ is constructed by means of toroidal embeddings ([14]). Then, for any point $q \in \mathscr{A}(m)-\mathscr{A}^{0}(m)$, there is a neighbourhood $V$ (resp. $U$ ) of $q$ in $\mathscr{A}(m)$ (resp. $\pi(q)$ in $S$ ) with $\pi(V)=U$ and local coordinates $\left\{u_{1}, u_{2}, \cdots, u_{n}\right\}$ in $U,\left\{v_{1}, v_{2}, \cdots, v_{(m+1) n}\right\}$ in $V$ such that on $S_{0}$ and $\mathscr{A}^{0}(m)$ we have

$$
\begin{aligned}
& u_{i}=\exp \left(2 \pi \sqrt{-1} \sum_{k} a_{i k} z_{k}\right) \\
& v_{j}=\prod_{k=1}^{n} u_{k}^{\alpha_{j k}} \prod_{k, l}\left(\exp 2 \pi \sqrt{-1} \zeta_{k}^{(l)}\right)^{\beta}{ }_{j k}^{(l)}
\end{aligned}
$$

where $a_{i k} \in R, \alpha_{j k}, \beta_{j k}^{(l)} \in Z$. Moreover, the morphism $\pi$ is given by

$$
u_{i}=\prod_{j=1}^{(m+1) n} v_{j}^{r_{i j}}
$$

where $\gamma_{i j}$ 's are non-negative integers. Then, in these coordinates, $d z_{1} \wedge$ $\cdots \wedge d z_{g} \wedge d \zeta_{N_{1}}^{(1)} \wedge \cdots \wedge d \zeta_{N_{m}}^{(m)}$ is expressed in a form

$$
\tau=\sum C_{k_{1} \cdots k_{d-n}} \prod_{i=1}^{n} \frac{d u_{i}}{u_{i}} \wedge \prod_{i=1}^{d-n} \frac{d v_{k_{i}}}{v_{k_{i}}}
$$

where $C_{k_{1} \cdots k_{d-n}}$ 's are constant. Note that, by (6.1),

$$
\prod_{i=1}^{n} d u_{i} \wedge \prod_{i=1}^{d-n} \frac{d v_{k_{i}}}{v_{k_{i}}}
$$

is holomorphic on $V$. Since $f$ is a cusp form,

$$
\frac{f}{u_{1} \cdots u_{n}} \sum C_{k_{1} \cdots k_{d-n}} \prod_{i=1}^{n} d u_{i} \wedge \prod_{i=1}^{d-n} \frac{d v_{k_{i}}}{v_{k_{i}}}
$$

is holomorphic on $V$.

Q.E.D.

Remark. From the above proof, it is clear that if $f$ is not a cusp form, $\omega_{f}$ can not be extended to a holomorphic form. 
The converse of the proposition is also valid. First we prove

PROPOSITION 2. Let $\omega=\sum f_{I J} d z_{I} \wedge d \zeta_{J_{1}}^{(1)} \wedge \cdots \wedge d \zeta_{J_{m}}^{(m)}$ be a G-invariant holomorphic $d$-form on $\mathscr{H}^{n} \times C^{m n}$. If $d<n$, then $\omega=0$. If $n \leq d \leq$ $(m+1) n$, then we have

$$
\omega=\sum g_{J} d z_{1} \wedge \cdots \wedge d z_{n} \wedge d \zeta_{J_{1}}^{(1)} \wedge \cdots \wedge d \zeta_{J_{m}}^{(m)},
$$

where $J=\left(J_{1}, \cdots, J_{m}\right)$, each $J_{i}$ is an ordered subset of $\{1,2, \cdots, n\}, \sum\left|J_{i}\right|$ $=d-n$ and $g_{J}$ is a Hilbert modular form of type $\left(k_{1}, k_{2}, \cdots, k_{n_{0}}\right)$ with $k_{i}$ $-2=\#\left\{J_{l} \mid i \in J_{l}\right\}$.

Proof. For simplicity we assume that $m=1$. The other case can be treated similarly and is left to the reader. We write

$$
\omega=\sum_{|I|=0}^{n}\left(\sum_{j} f_{I J} d z_{I} \wedge d \zeta_{J}\right)
$$

By a linear change of the coordinates $\left(\zeta_{1}, \cdots, \zeta_{g}\right)$, we can assume that the group $G$ acts on $\mathscr{H}^{n} \times \boldsymbol{C}^{n}$ in the form

$$
\begin{aligned}
& \left(z_{1}, \cdots, z_{n}, \zeta_{1}, \cdots, \zeta_{g}\right) \longrightarrow\left(\frac{a^{(1)} z_{1}+b^{(1)}}{c^{(1)} z_{1}+d^{(1)}}, \cdots, \frac{a^{(n)} z_{n}+b^{(n)}}{c^{(n)} z_{n}+d^{(n)}}, \tilde{\zeta}_{1}, \cdots, \tilde{\zeta}_{g}\right), \\
& \tilde{\zeta}_{i}=\left(c^{(i)} z_{i}+d^{(i)}\right)^{-1}\left(\zeta_{i}+\alpha^{(i)} z_{i}+\beta^{(i)}\right) .
\end{aligned}
$$

Consider now the translations

$$
\zeta_{i} \longrightarrow \zeta_{i}^{\prime}=\zeta_{i}+\alpha^{(i)} z_{i}+\beta^{(i)}
$$

on the fibres. Since $\omega$ is $G$-invariant, for the minimal value $e=|I|$ of $|I|$ with $f_{I J} \neq 0$, we have $f_{I J}(z, \zeta)=f_{I J}\left(z, \zeta^{\prime}\right)$. Hence, $f_{I J}$ with $|I|=e$ is constant along fibres. If $e<n$, for $f_{I^{\prime} J^{\prime}}$ with $\left|I^{\prime}\right|=e+1$, we obtain

$$
f_{I^{\prime} J^{\prime}}\left(z, \zeta^{\prime}\right)=f_{I^{\prime} J^{\prime}}(z, \zeta)+\sum_{|I|=e} m_{I J} f_{I J},
$$

where $m_{I J} \neq 0$ if $I \subset I^{\prime}, J^{\prime} \subset J$. In particular $\partial f_{I^{\prime} J^{\prime}} / \partial \zeta_{i}$ is invariant under the translations, hence constant along the fibres. Since $f_{I^{\prime} J^{\prime}}$ is invariant under translation $\zeta_{i} \rightarrow \zeta_{i}+\beta_{i}^{(i)}$, it follows that $f_{I^{\prime} J^{\prime}}$ is constant along the fibres. Hence, we have

$$
\sum_{|I|=e} m_{I J} f_{I J}=0
$$

The invariance of $\omega$ under $G$ implies

$$
f_{I J}(\gamma(z, \zeta))=\prod_{i=1}^{n}\left(c^{(i)} z_{i}+d^{(i)}\right)^{e_{i}(I, J)} f_{I J}(z, \zeta)
$$


where $e_{i}(I, J)=2 \cdot \sharp\{I \mid i \in I\}+\#\{J \mid i \in J\}$. Hence from (6.2) we infer that $f_{I J}=0$, if $e<n$ and $|J| \neq 0$. Hence, by induction, we arrive at the case $e=n$ or $e=d<n$. Then by (6.3), $f_{I J}$ is a Hilbert modular form of type $\left(e_{1}(I, J), \cdots, e_{n}(I, J)\right)$. It is well-known that if $e_{i}=0$ for some $i$, there is no non-zero Hilbert modular form of type $\left(e_{1}, \cdots, e_{n}\right)$.

Q.E.D.

By Propositions 1, 2 and the remark, we obtain

TheOREm. Let $\mathscr{A}(m)$ be any non-singular compactification of $\mathscr{A}^{\circ}(m)$. Then we have

$$
h^{0, d}\left(\mathscr{A}^{0}(m)\right)= \begin{cases}0, & \text { if } d<n \text { or } d>(m+1) n, \\
\sum_{\left(k_{1} \cdots k_{n}\right)}\left(\begin{array}{l}
m \\
k_{1}
\end{array}\right) \cdots\left(\begin{array}{c}
m \\
k_{n}
\end{array}\right) \operatorname{dim} S_{k_{1} \cdots k_{n}}, \quad \text { if } n \leq d \leq(m+1) n,\end{cases}
$$

where the sum is taken over all $n$-tuples $\left(k_{1}, \cdots, k_{n}\right)$ of integers satisfying

$$
k_{i} \geq 2, \quad \sum_{i=1}^{n} k_{i}=d+n .
$$

Corollary. Set $s\left(k_{1}, \cdots, k_{n}\right)=\operatorname{dim} S_{k_{1}, \cdots, k_{n}}$. Then the arithmetic genus $\chi\left(\mathscr{A}(m), \mathcal{O}_{\mathscr{A}(m)}\right)$ can be expressed as follows.

$$
\chi\left(\mathscr{A}(m), \mathcal{O}_{\mathscr{A}(m)}\right)=1+\sum_{d=n}^{(m+1) n} \sum_{\substack{\left(k_{1} \cdots k_{n}\right) \\
k_{i} \geq 2 \\
k_{i}=d+n}}(-1)^{d}\left(\begin{array}{l}
m \\
k_{1}
\end{array}\right) \cdots\left(\begin{array}{l}
m \\
k_{n}
\end{array}\right) s\left(k_{1}, \cdots, k_{n}\right) .
$$

\section{$\S_{1}$ 7. The arithmetic genus of $\mathscr{A}$}

We shall consider the fibre space $\tilde{\pi}: \tilde{\mathscr{A}}_{\frac{1}{2}}(\Gamma, \mathscr{M}) \rightarrow \tilde{S}(\Gamma)$ more carefully and compute its arithmetic genus. In this section we use the notations $\mathscr{A}=\tilde{\mathscr{A}}_{\frac{1}{2}}(\Gamma, \mathscr{M}), S=\tilde{S}(\Gamma), \pi=\tilde{\pi}: \mathscr{A} \rightarrow S$.

7.1. Since $\pi: \mathscr{A} \rightarrow S$ is flat, the dualising sheaf $\omega_{\mathscr{A} / S}$ of $\pi$ is isomorphic to $K_{A} \otimes \pi^{*} K_{S}^{-1}$, where $K_{X}$ denotes the canonical bundle of a complex manifold $X$.

Lemma 1. $\omega_{\mathscr{S} / S}=\pi^{*}(L)$ where $L$ is an invertible sheaf on $S$.

The proof is based on an explicit calculation of $K_{\mathscr{A}}$ by means of the non-singular compactification $\pi: \mathscr{A} \rightarrow S$ given in $\S 4$. The calculation is left to the reader. One can also use Proposition 1 in 6.1 .

Let $\mathcal{O}_{\mathscr{A}}$ be the structure sheaf of $\mathscr{A}$.

LEMMA 2. The sheaf $R^{k} \pi_{*} \mathcal{O}_{\star}$ is locally free for each $k \geq 0$. 
Proof. For each point $s \in S$, the fibre $A_{s}=\pi^{-1}(s)$ is reduced and Gorenstein. The dualising sheaf $\omega_{A_{s}}$ is isomorphic to $\omega_{\mathscr{S} / S} \otimes \mathcal{O}_{A_{s}}=\mathcal{O}_{A_{s}}$ by virtue of the above lemma. As $A_{s}$ is reduced, we have

$$
\begin{aligned}
& h^{0}\left(A_{s}, \mathcal{O}_{A_{s}}\right)=1 \\
& h^{2}\left(A_{s}, \mathcal{O}_{A_{s}}\right)=h^{0}\left(A_{s}, \omega_{A_{s}}\right)=1 .
\end{aligned}
$$

By the invariance of the Euler characteristic under flat deformations we obtain $h^{1}\left(A_{s}, \mathcal{O}_{A_{s}}\right)=2$.

Q.E.D.

Remark. We can directly calculate $H^{1}\left(A_{s}, \mathcal{O}_{A_{s}}\right)$ as follows. For each point $s \in S-\mathscr{H}^{2} / \Gamma$, the structure of $A_{s}$ can be seen from the cell decomposition $x \times \mathfrak{B}_{R}$ given in 4.7 and 4.8 and the action of $\mathcal{O}$ on this cell decomposition. Identify $x \times \mathfrak{B}_{R}$ with $\boldsymbol{R}^{2}$ and $\mathcal{O}$ with $Z^{2}$. Then the decomposition gives a cell decomposition of a real torus $T^{2}=R^{2} / Z^{2}$. To each 2-cell there corresponds an irreducible component of $A_{s}$ and to each 1-cell there corresponds an intersection curve of two components. Moreover from this decomposition we infer that each irreducible component is a non-singular rational surface and each intersection curve is a nonsingular rational curve. As was stated in 4.12, to the decomposition of $\mathfrak{B}_{R} \simeq \boldsymbol{R}^{2}$ there corresponds a fibre $X_{s}$ of the semi-universal covering $\mathscr{X}(\Gamma, \mathscr{M}) \rightarrow S$ over $s$. Then $A_{s}$ is the quotient of $X_{s}$ by the action of $\mathcal{O}$ $\widetilde{Z} Z^{2}$. (Note that the action is free.) Let us introduce an orientation on each cell in $R^{2}$ and write $Z_{\sigma}$ for the manifold corresponding to a cell $\sigma$. Then we have a resolution of the constant sheaf $Z_{X_{s}}$ on $X_{s}$

$$
0 \longrightarrow Z_{X_{s}} \stackrel{\iota^{*}}{\longrightarrow} \underset{\sigma, 2 \text {-cell }}{\bigoplus_{Z_{\sigma}}} \stackrel{\psi_{0}}{\longrightarrow} \underset{\tau, 1 \text {-cell }}{\bigoplus_{Z_{\tau}}} \stackrel{\psi_{1}}{\longrightarrow} \underset{\nu, 0 \text {-cell }}{\bigoplus_{Z_{\nu}}} Z_{Z} \longrightarrow 0
$$

by constant sheaves where $\iota^{*}$ is induced from the normalization"of $X_{s}$ and $\psi_{k}$ is given by

$$
\psi_{k}\left(\left(a_{\sigma}\right)\right)=\left(b_{\tau}\right) \quad b_{\tau}=\sum_{\tau<\sigma}[\sigma: \tau] a_{\sigma}
$$

Hence we have an exact sequence

$$
0 \longrightarrow \mathcal{O}_{X_{s}} \stackrel{\stackrel{\iota^{*}}{\longrightarrow}}{\longrightarrow} \oplus \mathcal{O}_{Z_{\sigma}} \stackrel{\psi_{0}}{\longrightarrow} \oplus \mathcal{O}_{Z_{\tau}} \stackrel{\psi_{1}}{\longrightarrow} \oplus \mathcal{O}_{Z_{\nu}} \longrightarrow 0
$$

Dividing by the action of $\mathcal{O}$, we obtain an exact sequence

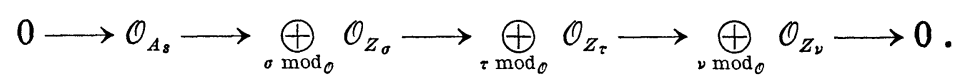


Since $Z_{\eta}$ is a non-singular rational variety for any cell $\eta$, we have $H^{p}\left(Z_{\eta}\right.$, $\left.\mathcal{O}_{z_{\eta}}\right)=0, p \geq 1$. From the exact sequence we have isomorphisms

$$
\begin{aligned}
H^{i}\left(A_{s}, \mathcal{O}_{A_{s}}\right) \simeq & H^{i}\left(\left\{\oplus H^{0}\left(Z_{\eta}, \mathcal{O}_{Z_{\eta}}\right)\right\}\right) \\
= & H^{i}\left(\left\{\oplus \boldsymbol{C}_{\eta}\right\}\right) \\
& \left.H^{i}\left(\boldsymbol{R}^{2} \mid \boldsymbol{Z}^{2}, C\right)=C{ }^{(2)}\right), \quad i=0,1,2 .
\end{aligned}
$$

Moreover, since $H^{1}\left(Z_{\eta}, Z\right)=0$ we have an isomorphism

$$
H^{1}\left(A_{s}, Z\right) \underset{Z}{\otimes} C \simeq H^{1}\left(A_{s}, \mathcal{O}_{A_{s}}\right) .
$$

This will be used later.

7.2. Let us consider the action of $\Gamma \subset S L_{2}\left(\mathcal{O}, \mathfrak{B}^{\prime}\right)$ (cf. 4.12). On $\mathscr{H}^{2}$ $\times \boldsymbol{C}^{2}$ defined by

$$
\begin{aligned}
\mathscr{H}^{2} \times C^{2} & \longrightarrow \mathscr{H}^{2} \times C^{2} \\
\Psi & \stackrel{\Psi}{\left.z_{1}, z_{2}, \zeta_{1}, \zeta_{2}\right)} \longrightarrow\left(\frac{\alpha^{(1)} z_{1}+\beta^{(1)}}{\gamma^{(1)} z_{1}+\delta^{(1)}}, \frac{\alpha^{(2)} z_{2}+\beta^{(2)}}{\gamma^{(2)} z_{2}+\delta^{(2)}},\left(\gamma^{(1)} z_{1}+\delta^{(1)}\right) \zeta_{1},\left(\gamma^{(2)} z_{2}+\delta^{(2)}\right) \zeta_{2}\right)
\end{aligned}
$$

for $\left(\begin{array}{ll}\alpha & \beta \\ \gamma & \delta\end{array}\right) \in \Gamma$. Then $\Gamma$ acts freely on $\mathscr{H}^{2} \times C^{2}$ and the quotient space $\mathscr{H}^{2} \times C^{2} / \Gamma$ with canonical projection to $S^{0}=\mathscr{H}^{2} / \Gamma$ defines a vector bundle of rank 2 over $S^{0}$. This bundle can be extended to $S$ as a holomorphic vector bundle.

To see this, let us recall briefly the compactification $S=\tilde{S}(\Gamma, \mathscr{M})$ of $S^{0}$. The isotropy group of $\Gamma$ at a cusp $\tau$ of $\bar{S}^{0}$ has the form

$$
G(N, V)=\left\{\left(\begin{array}{ll}
\varepsilon & \mu \\
0 & 1
\end{array}\right) \in S L_{2}(K) \mid \varepsilon \in V, \mu \in N\right\}
$$

(See 3.1). For a positive integer $d$, put $W(d)=\left\{\left(z_{1}, z_{2}\right) \in \mathscr{H}^{2} \mid \operatorname{Im} z_{1} \cdot \operatorname{Im} z_{2}\right.$ $>d\}$. Then for a sufficiently large $d, W(d) / G(N, V) \cup\{\tau\}$ is an open neighbourhood of the cusp $\tau$ of the Satake compactification of $S^{0}$. The quotient space $W(d) / N$ is an open set of $\left(C^{*}\right)^{2}$. Using the cone decomposition $\Sigma$ of $N_{\boldsymbol{R}}^{+}$which is a refinement of the Hirzebruch decomposition given in 3.1 , we construct a torus embedding $\mathscr{X}_{\Sigma}$ of $\left(C^{*}\right)^{2}$. Let $\mathscr{X}(d)$ be the interior of the closure $\overline{W(d) / N}$ in $\mathscr{X}_{\Sigma}$. Then $V$ operates freely on $\mathscr{X}(d)$ and $\mathscr{X}(d) / V$ gives a desingularization of the cusp $\tau$.

Now we extend our vector bundle to $\mathscr{X}(d) / V$. Since we have $W(d)$ 
$\times C^{2} / N=W(d) / N \times C^{2}$, this is the trivial bundle over $W(d) / N$. Hence it can be extended to $\mathscr{X}(d)$ as the trivial bundle $\mathscr{X}(d) \times C^{2}$. The group $V$ operates on $\mathscr{X}(d) \times C^{2}$ such that the action is the natural extension of that on $W(d) \times C^{2}$. The quotient $\mathscr{X}(d) \times C^{2} / V$ is a vector bundle over $\mathscr{X}(d) / V$. In this way we obtain the vector bundle $V_{\Gamma}$ over $S$ which is the extension of $\mathscr{H}^{2} \times C^{2} / \Gamma$ over $S^{0}=\mathscr{H}^{2} / \Gamma$.

Lemma 3. There is a natural isomorphism $R^{1} \pi_{*} \mathcal{O}_{\star} \widetilde{\mathcal{O}_{S}}\left(V_{\Gamma}\right)$.

Proof. First we construct an isomorphism over $S^{0}$. Put

$$
\omega_{i}=\bar{\partial}\left\{\left(\operatorname{Im} z_{i}\right)^{-1}\left(\zeta_{i}-\bar{\zeta}_{i}\right)\right\}, \quad i=1,2 .
$$

It is easy to see that $\omega_{1}, \omega_{2}$ define a trivialization of the sheaf $R^{1} \tilde{\pi}_{*} \mathcal{O}_{\tilde{\pi^{0}}}$ on $\mathscr{H}^{2}$ where $\tilde{\pi}: \tilde{\mathscr{A}}^{0} \rightarrow \mathscr{H}^{2}$ is the pull back of $\pi^{0}: \mathscr{A}^{0} \rightarrow S^{0}, \mathscr{A}^{0}=\pi^{-1}\left(S^{0}\right)$ over $\mathscr{H}^{2}$. We have

$$
\left(\begin{array}{l}
g^{*} \omega_{1} \\
g^{*} \omega_{2}
\end{array}\right)=\left(\begin{array}{l}
\left(\gamma^{(1)} z_{1}+\delta^{(1)}\right) \omega_{1} \\
\left(\gamma^{(2)} z_{2}+\delta^{(2)}\right) \omega_{2}
\end{array}\right), \quad g=\left(\begin{array}{ll}
\alpha & \beta \\
\gamma & \delta
\end{array}\right)
$$

Since $\left.R^{1} \pi_{*} \mathcal{O}_{\mathscr{A}}\right|_{S^{0}}$ is canonically isomorphic to $R^{1} \tilde{\pi}_{*} \mathcal{O}_{\tilde{\mathbb{A}^{0}}} / \Gamma$, there is a natural isomorphism

$$
\psi_{1}:\left.R^{q} \pi_{*} \mathcal{O}_{\mathscr{A}}\right|_{S^{0}} \stackrel{\sim}{\longrightarrow} \mathcal{O}_{S^{0}}\left(\left.V_{\Gamma}\right|_{S^{0}}\right) .
$$

Next, let us consider an open set $\mathscr{X}(d) / V$ described above. On $\mathscr{X}(d)$ we have a family $\varphi: \mathscr{A}^{\prime} \rightarrow \mathscr{X}(d)$ of semi-stable abelian varieties such that $\mathscr{A}^{\prime} / V \rightarrow \mathscr{X}(d) / V$ is the restriction of our family $\pi: \mathscr{A} \rightarrow S$ on $\mathscr{X}(d) / V$. By the remark given in 7.1 , we have a natural isomorphism

$$
\psi_{2}: R^{1} \varphi_{*}^{c} Z \underset{Z}{\otimes} \mathcal{O}_{\mathscr{A}_{c}} \stackrel{\sim}{\sim} R^{1} \varphi_{*}^{c} \mathcal{O}_{\otimes_{c}}
$$

where $\mathscr{X}(d)^{c}=\mathscr{X}(d)-W(d) / G, \varphi^{-1}\left(\mathscr{X}(d)^{c}\right)=\mathscr{A}_{c}, \varphi^{c}=\varphi \mid \mathscr{A}_{c}$. Now $R^{1} \varphi_{*}^{c} Z$ is trivial on $\mathscr{X}(d)^{c}$ and $V$ acts on it by multiplication by $\left(\varepsilon^{(1)^{-1}}, \varepsilon^{(2)-1}\right), \varepsilon \in V$. This means that there is a natural isomorphism

$$
\psi_{1}:\left.R^{1} \pi_{*} \mathcal{O}_{*}\right|_{D} \longrightarrow \mathcal{O}_{D}\left(\left.V_{\Gamma}\right|_{D}\right)
$$

in a neighbourhood $D$ of $\mathscr{X}(d)^{c} / V$ in $S$. It is easy to see that $\psi_{1}$ and $\psi_{2}$ are compatible on $(\mathscr{X}(d) / V) \cap S^{0}$.

Q.E.D.

Let $N$ be the normal bundle of $o(S)$ in $A$ where $o: S \rightarrow A$ is the zero section. It is easy to check that the vector bundle $N$ is isomorphic to $V_{\Gamma}$. Hence we have 
Corollary. $\quad R^{1} \pi_{*} \mathcal{O}_{*} \underset{\mathcal{O}}{\rightarrow}(N)$.

From this corollary we obtain

CoRollaRy. Let $\hat{\mathscr{A}}^{\sharp}$ be the analytic group scheme over $S$ defined by the exact sequence

$$
0 \longrightarrow R^{1} \pi_{*} Z \longrightarrow R^{1} \pi_{*} \mathcal{O}_{\triangleleft} \longrightarrow \mathcal{O}_{S}\left(\hat{\mathscr{A}}_{\#}\right) \longrightarrow 0
$$

where $\mathcal{O}_{S}\left(\hat{\mathscr{A}}_{\#}\right)$ is the sheaf of germs of locally holomorphic sections of $\hat{\mathscr{A}}^{\#}$. If $\Gamma$ admits a modular embedding into $S_{4}(Z)$ then $\hat{\mathscr{A}}_{\left.\#\right|_{S^{0}}}=\mathscr{A}^{0}$ and $\hat{\mathscr{A}}^{\sharp}$ is a dense open subset of $\mathscr{A}$. In general, $\hat{\pi}^{\#}:\left.\mathscr{A}^{\#}\right|_{S^{0}} \rightarrow S^{0}$ is a family of dual abelian varieties of $\pi: \mathscr{A}^{0} \rightarrow S^{0}$.

7.3. Let us consider actions of $\Gamma$ on $\mathscr{H}^{2} \times C$ defined by

$$
\begin{aligned}
\mathscr{H}^{2} \times C & \longrightarrow \mathscr{H}^{2} \times C \\
& \left(z_{1}, z_{2}, \zeta\right) \longrightarrow\left(\frac{\alpha^{(1)} z_{1}+\beta^{(1)}}{\gamma^{(1)} z_{1}+\delta^{(1)}}, \frac{\alpha^{(2)} z_{2}+\beta^{(2)}}{\gamma^{(2)} z_{2}+\delta^{(2)}},\left(\gamma^{(i)} z_{i}+\delta^{(i)}\right) \zeta\right),
\end{aligned}
$$

where $\left(\begin{array}{ll}\alpha & \beta \\ \gamma & \delta\end{array}\right) \in \Gamma$, and $i=1,2$. The quotient spaces of $\mathscr{H}^{2} \times C$ by these actions are complex line bundles $L_{0}^{(i)}$ on $S^{0}$. By an argument similar to the one used above, $L_{0}^{(i)}$ can be extended to $S$ as a complex line bundle $L_{i}$. From the definition of $V_{\Gamma}$ we have $V_{\Gamma}=L_{1} \oplus L_{2}$.

By an argument as used in the proof of Lemma 3, we can prove the following lemma.

Lemma 4. There is a natural isomorphism

$$
R^{2} \pi_{*} \mathcal{O}_{\mathscr{A}} \stackrel{\longrightarrow}{\longrightarrow} \mathcal{O}_{S}\left(\wedge^{2} V_{\Gamma}\right)
$$

On the other hand, by the Grothendieck duality for the morphism $\pi$, $\pi_{*} \omega_{\mathscr{s} / S}$ is dual to $R^{2} \pi_{*} \mathcal{O}_{\mathscr{\alpha}}$. Hence we have

Corollary. The line bundle $L$ in Lemma 1 is dual to $\wedge^{2} V_{\Gamma}$.

Remark. The vector space $H^{0}\left(S,\left(\wedge^{2} V_{\Gamma}^{\curlyvee}\right)^{\otimes m}\right)=H^{0}\left(S, L^{\otimes m}\right)$ is the vector space of modular forms of type $(m, m)$.

LEMma 5. The spectral sequence

$$
E_{2}^{p, q}=H^{p}\left(S, R^{q} \pi_{*} \mathcal{O}_{\mathscr{A}}\right) \Longrightarrow H^{p+q}\left(\mathscr{A}, \mathscr{A}_{\mathscr{A}}\right)
$$

degenerates at $E_{2}$-terms. 
Proof. Define, on a regular fibre of $\pi: \mathscr{A} \rightarrow S$, the multiplication by a positive integer $m$. This gives a morphism $m: \mathscr{A}^{0} \rightarrow \mathscr{A}^{0}$ and a meromorphic mapping of $\mathscr{A}$. By a succession of blowing ups with non-singular centres, we obtain a morphism $m^{\prime}: \mathscr{A}^{\prime} \rightarrow \mathscr{A}$. Since $R^{k} \psi_{*} \mathcal{O}_{\mathscr{A}}=0$ for $k>1$ and $\psi_{*} \mathcal{O}_{\mathscr{A}^{\prime}}=\mathcal{O}_{\mathscr{A}}$ for the morphism $\psi: \mathscr{A}^{\prime} \rightarrow \mathscr{A}$, there is a canonical iso-

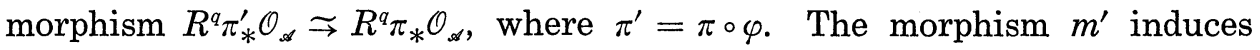
an endomorphism of $R^{q} \pi_{*} \mathcal{O}_{\mathscr{A}}$, which is multiplication by $m^{q}$. The morphism $m^{\prime}$ induces an endomorphism of the spectral sequence such that it commutes with $d_{2}^{p, q}$ and on $E_{2}^{p, q}$ it is multiplication by $m^{q}$. Hence $d_{2}$ must be zero.

Q.E.D.

Proposition. We have the following isomorphisms:

$$
\begin{aligned}
& H^{4}\left(\mathscr{A}, \mathcal{O}_{\mathscr{A}}\right) \stackrel{\sim}{\longrightarrow} H^{2}\left(S, R^{2} \pi_{*} \mathcal{O}_{\mathscr{A}}\right), \\
& H^{3}\left(\mathscr{A}, \mathcal{O}_{\mathscr{A}}\right) \sim H^{2}\left(S, R^{1} \pi_{*} \mathcal{O}_{\mathscr{A}}\right), \\
& H^{2}\left(\mathscr{A}, \mathcal{O}_{\mathscr{A}}\right) \stackrel{\sim}{\longrightarrow} H^{2}\left(S, \mathcal{O}_{\mathscr{A}}\right) \\
& H^{1}\left(\mathscr{A}, \mathcal{O}_{\mathscr{A}}\right)=0 .
\end{aligned}
$$

Proof. By Lemma 5, we have

$$
h^{p}\left(\mathscr{A}, \mathcal{O}_{\mathscr{A}}\right)=\sum_{i=0}^{2} \operatorname{dim} H^{p-i}\left(S, R^{i} \pi_{*} \mathcal{O}_{\mathscr{A}}\right) .
$$

$H^{2}\left(S, R^{1} \pi_{*} \mathcal{O}_{\&}\right)$ is dual to $H^{0}\left(S, K_{S} \otimes\left(R^{1} \pi_{*} \mathcal{O}_{s}\right)^{\vee}\right)$. By the definition of $L_{i}$, it is easy to see that $H^{0}\left(S, K_{S} \otimes L_{1}^{-1}\right)$ (resp. $H^{0}\left(S, K_{S} \otimes L_{2}^{-1}\right)$ ) is the space of modular forms of type $(3,2)$ (resp. type $(2,3)$ ). Hence by Theorem in 6.3,

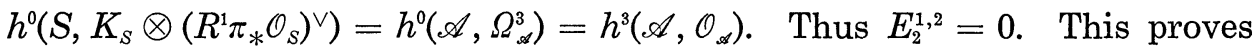
the first three isomorphisms. On the other hand, since $\mathscr{A}$ is simply connected we have $h^{1}\left(\mathscr{A}, \mathcal{O}_{\mathscr{A}}\right)=0$.

Q.E.D.

Corollary. $\quad H^{p}\left(S, R^{1} \pi_{*} \mathcal{O}_{\mathscr{A}}\right)=0$ for $p \leq 1$.

Now let us compute the arithmetic genus $\chi\left(\mathscr{A}, \mathcal{O}_{\mathscr{S}}\right)$. By the above spectral sequence, we have

$$
\begin{aligned}
\chi\left(\mathscr{A}, \mathcal{O}_{\mathscr{A}}\right) & =\sum(-1)^{p+q} \operatorname{dim} E_{2}^{p, q} \\
& =\chi\left(S, \mathcal{O}_{S}\right)-\chi\left(S, R^{1} \pi_{*} \mathcal{O}_{\mathscr{A}}\right)+\chi\left(S, R^{2} \pi_{*} \mathcal{O}_{\mathscr{A}}\right) \\
& =\chi\left(S, \mathcal{O}_{S}\right)-\chi\left(S, \mathcal{O}_{S}\left(V_{\Gamma}\right)\right)+\chi\left(S, \mathcal{O}_{S}\left(L^{-1}\right)\right) .
\end{aligned}
$$

By the Hirzebruch Riemann-Roch theorem, we have

$$
\begin{aligned}
& \chi\left(S, V_{\Gamma}\right)=\frac{1}{6}\left(c_{1}^{2}+c_{2}\right)+\frac{1}{2} c_{1} \cdot c_{1}\left(V_{\Gamma}\right)+\frac{1}{12}\left(c_{1}\left(V_{\Gamma}\right)^{2}-2 c_{2}\left(V_{\Gamma}\right)\right), \\
& \chi\left(S, \mathcal{O}_{S}\left(L^{-1}\right)\right)=\frac{1}{2} L \cdot\left(L+K_{S}\right)+\chi\left(S, \mathcal{O}_{\&}\right),
\end{aligned}
$$


where $c_{1}, c_{2}$ are the Chern classes of $S$. Since $V_{\Gamma}$ is isomorphic to $L_{1} \oplus$ $L_{2}, V_{T}$ satisfies the condition of Mumford's theorem on the Hirzebruch proportionality principle in the non-compact case ([12], p. 262). Indeed, $V_{T}$ corresponds to $\mathcal{O}_{P_{1}}(1) \oplus \mathcal{O}_{P^{1}}(1)$ over $\boldsymbol{P}^{1} \times \boldsymbol{P}^{1}$ which is the compact dual of $\mathscr{H}^{2}$. Hence $c_{1}\left(V_{\Gamma}\right)^{2}=2 c_{2}\left(V_{\Gamma}\right)$. Moreover, by Corollary to Lemma 4 , $c_{1}\left(V_{\Gamma}\right) \cdot c_{1}=L \cdot K_{S}$. Hence we have

$$
\chi\left(\mathscr{A}, \mathcal{O}_{\mathscr{A}}\right)=2 \chi\left(S, \mathcal{O}_{S}\right)-\frac{1}{6}\left(c_{1}^{2}+c_{2}\right)+\frac{1}{2} L^{2}=\frac{1}{2} L^{2},
$$

since $\chi\left(S, \mathcal{O}_{S}\right)=\frac{1}{12}\left(c_{1}^{2}+c_{2}\right)$. On the other hand, $H^{0}\left(S, L^{-m}\right)$ is the vector space of modular forms of type $(m, m)$. Hence, by the Riemann-Roch theorem, we have

$$
\frac{1}{2} L^{2}=\lim _{m \rightarrow+\infty} \frac{1}{m^{2}} h^{0}\left(S, L^{-m}\right)
$$

Moreover, it is easy to see that

$$
\lim _{m \rightarrow+\infty} \frac{1}{m^{2}} h^{0}\left(S, L^{-m}\right)=\lim _{m \rightarrow+\infty} \frac{1}{m^{2}} \operatorname{dim} S_{m, m}
$$

where $S_{m, m}$ is the space of cusp forms of type $(m, m)$. By Shimizu's dimension formula (see the remark in 3.3) we obtain

$$
\frac{1}{2} L^{2}=\operatorname{vol}\left(\mathscr{H}^{2} / \Gamma\right) \text {. }
$$

Theorem. $\quad \chi\left(\mathscr{A}, \mathcal{O}_{\mathscr{A}}\right)=\operatorname{vol}\left(\mathscr{H}^{2} / \Gamma\right)$, where we use the volume form

$$
\frac{1}{4(2 \pi)^{2}} \prod_{i=1}^{2} \frac{d z_{i} \wedge d \bar{z}_{i}}{\left(\operatorname{Im} z_{i}\right)^{2}} \quad \text { on } \mathscr{H}^{2}
$$

Remark. Such a theorem also holds for elliptic modular surfaces. One can obtain a similar result from Shioda's formula for the invariants of elliptic modular surface ([15]) in that case.

\section{$\S 8$. On the number of sections of $\mathscr{A}(\Gamma, \mathscr{M})$}

The aim of this section is to prove that the families of abelian surfaces constructed in 1.10 and 1.11 admit only finitely many holomorphic sections.

8.1. Let $\varphi: \mathscr{A} \rightarrow \mathscr{V}=\mathscr{H} / G$ be a family of abelian varieties over the curve $\mathscr{V}$ obtained from the action of $G \times \mathscr{M}\left(\mathscr{M}\right.$ a lattice in $\left.C^{n}\right)$ on $\mathscr{H}$ $\times \boldsymbol{C}^{n}$. We assume that $G$ is an arithmetic subgroup of $S L_{2}(R)$ which 
acts properly discontinuously and freely on $\mathscr{H}$ such that $\mathscr{V}=\mathscr{H} / G$ is compact. Then $R^{k} \varphi_{*} \mathcal{O}_{\star}(k \geq 0)$ is a locally free sheaf on $\mathscr{V}$. We claim:

$$
\operatorname{rank} H^{1}\left(\mathscr{V}, R^{1} \varphi_{*} Z\right)=2 \operatorname{dim} H^{1}\left(\mathscr{V}, R^{1} \varphi_{*} \mathcal{O}_{⿻}\right) .
$$

We give a proof without appealing to the theory of vector valued differential forms.

Consider the two exact sequences

$$
0 \longrightarrow \varphi_{*} \Omega_{\star / /} \longrightarrow R^{1} \varphi_{*} C \otimes \mathcal{O}_{r} \longrightarrow R^{1} \varphi_{*} \mathcal{O}_{\star} \longrightarrow 0
$$

and

$$
0 \longrightarrow R^{1} \varphi_{*} C \longrightarrow R^{1} \varphi_{*} C \otimes \mathcal{O}_{\digamma} \stackrel{\nabla}{\longrightarrow} \Omega_{\digamma}^{1} \otimes R^{1} \varphi_{*} C \longrightarrow \Omega_{\curlyvee}^{2} \otimes \varphi_{*} C \longrightarrow \cdots,
$$

where $\nabla$ is the Gauss-Manin connection. Since $\operatorname{dim} V=1, \Omega_{r}^{2} \otimes R^{1} \varphi_{*} C$ $=0$ and we get

$$
\begin{aligned}
& 0 \longrightarrow H^{0}\left(\mathscr{V}, R^{1} \varphi_{*} C\right) \longrightarrow H^{0}\left(\mathscr{V}, R^{1} \varphi_{*} C \otimes \mathcal{O}_{\mathscr{r}}\right) \longrightarrow H^{0}\left(\mathscr{V}, R^{1} \varphi_{*} C \otimes \Omega_{\mathscr{r}}^{1}\right) \\
& \longrightarrow H^{1}\left(\mathscr{V}, R^{1} \varphi_{*} C\right) \longrightarrow H^{1}\left(\mathscr{V}, R^{1} \varphi_{*} C \otimes \mathcal{O}_{\curlyvee}\right) \longrightarrow H^{1}\left(\mathscr{V}, R^{1} \varphi_{*} C \otimes \Omega_{\mathscr{V}}^{1}\right) \\
& \longrightarrow H^{2}\left(\mathscr{V}, R^{1} \varphi_{*} C\right) \longrightarrow 0 .
\end{aligned}
$$

Now $R^{1} \varphi_{*} C$ is locally constant and self-dual (use the Riemann form), hence under duality this sequence goes over into itself. In order to prove our claim we must show

$$
\operatorname{dim}\left(\text { image } H^{0}\left(\mathscr{V}, \Omega_{\mathscr{r}}^{1} \otimes R^{1} \varphi_{*} C\right) \longrightarrow H^{1}\left(\mathscr{V}, R^{1} \varphi_{*} C\right)\right)=\operatorname{dim} H^{1}\left(\mathscr{V}, R^{1} \varphi_{*} \mathcal{O}_{\mathscr{A}}\right)
$$

and

$$
\operatorname{dim}\left(\text { image } H^{1}\left(\mathscr{V}, R^{1} \varphi_{*} C\right) \longrightarrow H^{1}\left(\mathscr{V}, R^{1} \varphi_{*} C \otimes \mathcal{O}_{\mathscr{r}}\right)\right)=\operatorname{dim} H^{1}\left(\mathscr{V}, R^{1} \varphi_{*} \mathcal{O}_{\Im}\right)
$$

But by duality the two statements are equivalent. We shall prove the first. It is not difficult to show that $H^{0}\left(\mathscr{V}, R^{1} \varphi_{*} \mathcal{O}_{r}\right)=0$. Consequently, we obtain from $(*)$

$$
\begin{aligned}
& 0 \longrightarrow H^{0}\left(\mathscr{V}, \varphi_{*} \Omega_{\mathscr{\alpha} \mid \mathscr{\gamma}}^{1}\right) \longrightarrow H^{0}\left(\mathscr{V}, R^{1} \varphi_{*} C \otimes \mathcal{O}_{\mathscr{r}}\right) \longrightarrow 0 \\
& 0 \longrightarrow H^{1}\left(\mathscr{V}, \varphi_{*} \Omega_{\mathscr{*} \mid \mathscr{\gamma}}^{1}\right) \longrightarrow H^{1}\left(\mathscr{V}, R^{1} \varphi_{*} C \otimes \mathcal{O}_{r}\right) \longrightarrow H^{1}\left(\mathscr{V}, R^{1} \varphi_{*} \mathcal{O}_{\mathscr{A}}\right) \longrightarrow 0 \text {. }
\end{aligned}
$$

Hence

$$
\begin{aligned}
& \operatorname{dim} H^{0}\left(\mathscr{V}, R^{1} \varphi_{*} C \otimes \Omega_{\mathscr{r}}^{1}\right)-\operatorname{dim} H^{0}\left(\mathscr{V}, R^{1} \varphi_{*} C \otimes \mathcal{O}_{\curlyvee}\right) \\
& =\operatorname{dim} H^{1}\left(\mathscr{V}, R^{1} \varphi_{*} C \otimes \mathcal{O}_{r}\right)-\operatorname{dim} H^{0}\left(\mathscr{V}, \varphi_{*} \Omega_{\mathscr{A} \mid r}^{1}\right) \\
& =\operatorname{dim} H^{1}\left(\mathscr{V}, \varphi_{*} \Omega_{\mathscr{A} \mid \mathscr{V}}^{1}\right)+\operatorname{dim} H^{1}\left(\mathscr{V}, R^{1} \varphi_{*} \mathcal{O}_{\mathscr{A}}\right)-\operatorname{dim} H^{0}\left(\mathscr{V}, \varphi_{*} \Omega_{\mathscr{A} \mid \mathscr{V}}^{1}\right) \\
& =\operatorname{dim} H^{1}\left(\mathscr{V}, R^{1} \varphi_{*} \mathcal{O}_{\mathscr{A}}\right)
\end{aligned}
$$


since $\operatorname{dim} H^{1}\left(\mathscr{V}, \varphi_{*} \Omega_{\mathscr{S} \mid \mathscr{\gamma}}^{1}\right)=\operatorname{dim} H^{0}\left(\mathscr{V},\left(\varphi_{*} \Omega_{\mathscr{S} \mid \gamma}^{1}\right)^{\vee} \otimes \Omega_{\gamma}^{1}\right)$ and by a local computation $\left(\varphi_{*} \Omega_{\mathscr{A} \mid \boldsymbol{r}}^{1}\right)^{\vee} \otimes \Omega_{\mathscr{r}}^{1} \cong \varphi_{*} \Omega_{\mathscr{A} \mid \boldsymbol{r}}^{1}$. From this our claim follows.

Now let $\mathscr{A}^{\#}$ be the group scheme associated to $\varphi: \mathscr{A} \rightarrow \mathscr{V}$. Denote by $\mathscr{A}^{\#}$ the dual of $\mathscr{A}^{\#}$. Then we have an exact sequence

$$
0 \longrightarrow R^{1} \varphi_{*} Z \longrightarrow R^{1} \varphi_{*} \mathcal{O}_{s} \longrightarrow \mathcal{O}\left(\hat{\mathscr{A}}^{\#}\right) \longrightarrow 0 \text {. }
$$

Since $H^{0}\left(\mathscr{V}, R^{1} \varphi_{*} \mathcal{O}_{\mathscr{A}}\right)=0$, we get

$$
\begin{aligned}
0 \longrightarrow H^{0}\left(\mathscr{V}, \mathcal{O}\left(\hat{\mathscr{A}}^{*}\right)\right) \longrightarrow H^{1}\left(\mathscr{V}, R^{1} \varphi_{*} Z\right) \longrightarrow H^{1}\left(\mathscr{V}, R^{1} \varphi_{*} \mathcal{O}_{\&}\right) \longrightarrow \\
\quad \longrightarrow H^{1}\left(\mathscr{V}, \mathcal{O}\left(\hat{\mathscr{A}}^{*}\right)\right) \longrightarrow H^{2}\left(\mathscr{V}, R^{1} \varphi_{*} Z\right) \longrightarrow H^{2}\left(\mathscr{V}, R^{1} \varphi_{*} \mathcal{O}_{\&}\right) \longrightarrow \cdots
\end{aligned}
$$

Consider the usual exact sequence

$$
0 \longrightarrow Z \longrightarrow \mathcal{O}_{*} \longrightarrow \mathcal{O}_{*}^{*} \longrightarrow 0 \text {. }
$$

The homomorphism $H^{1}\left(\mathscr{V}, R^{1} \varphi_{*} Z\right) \rightarrow H^{1}\left(\mathscr{V}, R^{1} \varphi_{*} \mathcal{O}_{\mathscr{A}}\right)$ is the restriction of the homomorphism $H^{2}(\mathscr{A}, Z) \rightarrow H^{2}\left(\mathscr{A}, \mathcal{O}_{\mathscr{A}}\right)$ (induced by this short exact sequence) to the part of the cohomology on which multiplication by $m$ in the fibre induces multiplication by $m$. As is well-known, $H^{2}(\mathscr{A}, Z)$ generates $H^{2}\left(\mathscr{A}, \mathcal{O}_{\mathscr{A}}\right)$ over $\boldsymbol{R}$. Hence, since $\operatorname{dim} H^{2}\left(\mathscr{A}, \mathcal{O}_{\mathscr{A}}\right)=\operatorname{dim} H^{1}\left(\mathscr{V}, R^{1} \varphi_{*} \mathcal{O}_{\mathscr{A}}\right)=$ 2 rank $H^{1}\left(\mathscr{V}, R^{1} \varphi_{*} Z\right)$, it follows that $H^{0}\left(\mathscr{V}, \mathcal{O}\left(\hat{\mathscr{A}}^{\sharp}\right)\right)$ has to be finite. Using the dual family, it follows that $H^{0}\left(\mathscr{V}, \mathcal{O}\left(\mathscr{A}^{\sharp}\right)\right)$ is also finite. We have thus proved:

Proposition. The fibre space $\varphi: \mathscr{A} \rightarrow \mathscr{V}$ of abelian varieties over $\mathscr{V}$ $=\mathscr{H} / G$ possesses only finitely many holomorphic sections.

8.2. Assume now that $\Gamma$ is again a Hilbert modular group of a real quadratic field $K$ without elements of finite order. Let $\pi: \mathscr{A}(\Gamma, \mathscr{M}) \rightarrow S(\Gamma)$ be any toroidal compactifiaction of $\pi^{0}: \mathscr{A}^{0}(\Gamma, \mathscr{M}) \rightarrow \mathscr{H}^{2} / \Gamma$, a family of abelian surfaces with real multiplication, obtained from the action of $\Gamma \times \mathscr{M}$ on $\mathscr{H}^{2} \times C^{2}$. The image of $\mathscr{H}^{2}$ under the period map $\mathscr{H}^{2} \rightarrow \mathfrak{S}_{2}$ intersects infinitely many subvarieties of $\widetilde{S}_{2}$ given by singular relations (see 1.4). These intersections determine curves which are given in $\mathscr{H}^{2}$ by equations of the form:

$$
\alpha z_{1} z_{2}+\beta^{(1)} z_{1}+\beta^{(2)} z_{2}+\gamma=0 \quad(\alpha, \gamma \in Z, \beta \in K) .
$$

These curves (or their quotients under $\mathscr{H}^{2} \rightarrow \mathscr{H}^{2} / \Gamma$ ) are just the curves " $F_{N}$ " studied by Hirzebruch (cf. [8]). There are infinitely many curves $F_{N}$ which are compact in $\mathscr{H}^{2} / \Gamma$, and they lie dense in $\mathscr{H}^{2} / \Gamma$. Each compact curve is the quotient of $\mathscr{H}$ by a unit group $\Gamma_{1}$ of an order in a division 
quaternion algebra. Let $\mathscr{A}_{0}^{*}$ be the identity component of the group scheme associated to $\mathscr{A}$.

THEOREM. Let $\Gamma$ be a congruence subgroup of the Hilbert modular group $S L_{2}(\mathcal{O}, a)$ and let $\pi: \mathscr{A} \rightarrow S$ be a toroidal compactification of a family of abelian surfaces $\mathscr{A}^{0}=\mathscr{H}^{2} \times C^{2} / \Gamma \times \mathscr{M}$. Then $H^{0}\left(\mathscr{H}^{2} / \Gamma, \mathcal{O}\left(\mathscr{A}_{0}^{\sharp}\right)\right)$ is finite.

Proof. We may assume that $\Gamma$ is a principal congruence subgroup. Then $\pi^{0}: \mathscr{A}^{0} \rightarrow{ }^{2} / \Gamma$ carries a level $\mathfrak{B}$ structure (B an ideal). Let $\sigma$ be a section of $\mathscr{A}|\mathscr{H}| \Gamma_{1}$. Pull it back to $\mathscr{H} \times C^{2}$. Since it is of finite order it is of the form $\alpha z+\beta, \alpha, \beta \in K$ and $(\alpha \beta)\left(\gamma^{-1}\right) \in \mathcal{O} \oplus \mathfrak{a}$ for all $\gamma \in \Gamma_{1}$. Since $\gamma \equiv 1(\bmod \mathfrak{b})$ it follows that $\mathrm{N}(\mathfrak{b}) \sigma=0$. Since $H^{0}\left(\mathscr{H}^{2} / \Gamma, \mathcal{O}\left(\mathscr{A}_{0}^{\sharp}\right)\right)$ is finitely generated, this proves the Theorem, for $N(\mathfrak{B}) \sigma$ vanishes on a dense subset of $\mathscr{H}^{2} / \Gamma$ for all sections $\sigma$.

Remark. If $\Gamma$ is the principal congruence subgroup of level $\mathfrak{A}$ in $S L_{2}(\mathcal{O}, \mathfrak{B})$ then the $N(\mathfrak{U})^{2} \mathfrak{Y}$-section points define sections of $\mathscr{A}^{0}$ that can be extended to the toroidal compactification defined in $\S 4$ and these give all sections of $\mathscr{A}_{0}^{\#}$.

Remark. By applying a similar reasoning to families of abelian surfaces over $\widetilde{S}^{2} / \Gamma(m)$, where $\Gamma(m)$ is the level $m$ congruence group of $S p_{4}(Z, D)$, we obtain an analogous theorem in this case.

8.3. Let $\Gamma$ be a congruence subgroup of a Hilbert modular group which acts freely on $\mathscr{H}^{2}$ and denote the family of stable abelian varieties for $\Gamma$ constructed in section 4 by $\pi: \mathscr{A} \rightarrow S$. We then have the exact sequence

$$
0 \longrightarrow R^{1} \pi_{*} Z \longrightarrow R^{1} \pi_{*} \mathcal{O}_{*} \longrightarrow \mathcal{O}\left(\hat{A}_{0}^{\#}\right) \longrightarrow 0,
$$

where $\mathscr{A}_{0}^{\#}$ is the identity component of the dual group scheme of $\mathscr{A}$. It gives rise to the exact sequence

$$
\begin{aligned}
& \longrightarrow H^{1}\left(S, R^{1} \pi_{*} \mathcal{O}_{\mathscr{A}}\right) \longrightarrow H^{1}\left(S, \mathcal{O}\left(\hat{\mathscr{A}}_{0}^{\sharp}\right)\right) \longrightarrow H^{2}\left(S, R^{1} \pi_{*} Z\right) \\
& \longrightarrow H^{2}\left(S, R^{1} \pi_{*} \mathcal{O}_{\mathscr{A}}\right) \longrightarrow \cdots
\end{aligned}
$$

In section 6 we proved that $\operatorname{dim} H^{1}\left(S, R^{1} \pi_{*} \mathcal{O}_{\mathscr{A}}\right)=0$. This implies that $H^{1}\left(S, \mathcal{O}\left(\hat{\mathscr{A}}_{0}^{\sharp}\right)\right)$ is a discrete $\mathcal{O}$-module. To each element $\eta \in H^{1}\left(S, \mathcal{O}\left(\mathscr{A}_{0}^{\sharp}\right)\right)$ given by the cocycle $\left\{\eta_{i j}\right\}$ on the covering $\left\{U_{i}\right\}$ of $S$ we associate the analytic fibre space obtained by gluing $\left.\mathscr{A}\right|_{U_{i}}$ and $\left.\mathscr{A}\right|_{U_{j}}$ over $U_{i} \cap U_{j}$ by means of the translation in the fibre given by $\eta_{i j}$. The analytic space $\mathscr{A}^{n}$ thus 
obtained depends up to isomorphism only on the cohomology class of $\left\{\eta_{i j}\right\}$. An element $\alpha \in \mathcal{O}$ determines a holomorphic map $\psi_{\alpha}: \mathscr{A}^{\eta} \rightarrow \mathscr{A}^{\alpha \eta}$. From this it follows that if $\eta$ is of finite order then $A^{\eta}$ is an algebraic fourfold. We can also prove the converse. It is therefore of some interest to determine the structure of $H^{1}\left(S, \mathcal{O}\left(\mathscr{A}_{0}^{*}\right)\right)$.

\section{BIBLIOGRAPHY}

[1] Ash, A., Mumford, D., Rapoport, M. and Tai, Y., Smooth compactification of locally symmetric varieties, Math. Sci. Press, Brookline, 1975.

[2] Baily, W. and Borel, A., Compactification of arithmetic quotients of bounded symmetric domains, Ann. of Math., 84 (1966), 442-528.

[ 3 ] Freitag, E. and Schneider, V., Bemerkung zu einem Satz von J. Igusa und W. Hammond, Math. Z., 102 (1967), 9-16.

[4] Hammond, W., The modular groups of Hilbert and Siegel, Amer. J. Math., 88 (1966), 497-516.

[5] — The two actions of Hilbert's modular group, Amer. J. Math., 99 (1977), 389-392.

[6 ] Hecke, E., Höhere Modulfunktionen und ihre Anwendung auf die Zahlentheorie, Math. Ann., 71 (1912), 1-37.

[ 7 ] Hirzebruch, F., Hilbert modular surfaces, Enseign. Math., 71 (1973), 183-281.

[ 8 ] Hirzebruch, F. and Zagier, D., Intersection numbers of curves on Hilbert modular surfaces and modular forms of Nebentypus, Invent. Math., 36 (1976), 57-113.

[9] Humbert, G., Sur les fonctions abéliennes singulières I-III, J. de Math., série 5, tome V (1899), 233-350, tome VI (1900), 279-386, tome VII (1901), 97-123.

[10] Kempf, G., Knudsen, F., Mumford, D. and Saint-Donat, B., Toroidal embeddings I, Lect. Notes in Math. 339, Springer, Berlin-Heidelberg-New York, 1970.

[11] Mumford, D., An analytic construction of degenerating abelian varieties over complete rings, Comp. Math., 24 (1972), 239-272.

[12] - Hirzebruch's proportionality theorem in the non-compact case, Invent. Math., 42 (1977), 239-272.

[13] Namikawa, Y., A new compactification of the Siegel space and degeneration of abelian varieties I, II, Math. Ann., 221 (1976), 97-141, 201-241.

[14] — , Toroidal degeneration of abelian varieties II. Math. Ann. 245 (1979), 117150.

[15] Shioda, T., On elliptic modular surfaces, J. Math. Soc. Japan, 24 (1972), 20-59.

[16] Svarčman, O., Simple connectedness of the factor space of the Hilbert modular group (in Russian), Functional Anal. Appl., 8 (1974), 99-100.

University of Amsterdam

Kyoto University 